\title{
GET RID OF UNANIMITY: \\ THE SUPERIORITY OF MAJORITY RULE WITH VETO POWER
}

\author{
Laurent Bouton \\ Aniol Llorente-Saguer \\ Frédéric Malherbe \\ Working Paper 20417 \\ http://www.nber.org/papers/w20417 \\ NATIONAL BUREAU OF ECONOMIC RESEARCH \\ 1050 Massachusetts Avenue \\ Cambridge, MA 02138 \\ August 2014
}

We thank participants to the NDAM 2013 conference, the Columbia Political Economy Conference 2013, the Priorat Political Economy Workshop 2013, the Barcelona GSE PhD Jamboree 2014, the Workshop "Theory Meets Experiments" in Zurich, the Workshop on IIBEO in Alghero and seminar participants at Ben Gurion University, Boston University, London Business School, the Max Planck Institute in Bonn, New Economic School, New York University (NY and AD), Technion and University of Haifa. We particularly thank David Austen-Smith, Jean-Pierre Benoit, Micael Castanheira, Ignacio Esponda, Jean-Guillaume Forand, David Myatt, Roger Myerson, and Wolfgang Pesendorfer. The views expressed herein are those of the authors and do not necessarily reflect the views of the National Bureau of Economic Research.

NBER working papers are circulated for discussion and comment purposes. They have not been peerreviewed or been subject to the review by the NBER Board of Directors that accompanies official NBER publications.

(C) 2014 by Laurent Bouton, Aniol Llorente-Saguer, and Frédéric Malherbe. All rights reserved. Short sections of text, not to exceed two paragraphs, may be quoted without explicit permission provided that full credit, including $(0$ notice, is given to the source. 
Get Rid of Unanimity: The Superiority of Majority Rule with Veto Power

Laurent Bouton, Aniol Llorente-Saguer, and Frédéric Malherbe

NBER Working Paper No. 20417

August 2014

JEL No. D70

\section{ABSTRACT}

A group of agents wants to reform the status quo if and only if this is Pareto improving. Agents have private information and may have common or private objectives, which creates a tension between information aggregation and minority protection. We analyze a simple voting system - majority rule with veto power (Veto) - that essentially resolves this tension, for it combines the advantageous properties of both majority and unanimity rules. We argue that our results shed new light on the evolution of voting rules in the EU institutions and could help to inform debates about policy reforms in cases such as juries in the US.

Laurent Bouton

Georgetown University

Department of Economics

37th \& O Streets, NW

Washington,DC 20057

and NBER

boutonllj@gmail.com

Aniol Llorente-Saguer

School of Economics and Finance

Queen Mary, University of London

Mile End Road, London E1 4NS

United Kingdom

a.llorente-saguer@qmul.ac.uk
Frédéric Malherbe

London Business School

Regent's Park

NW1 4SA London

United Kingdom

fmalherbe@london.edu 


\section{Introduction}

A group of agents wants to reform the status quo if and only if this is Pareto improving. What voting system should they use? An obvious candidate is unanimity rule, which has been described as the incarnation of the Pareto criterion (Wicksell 1967 [1896], Buchanan and Tullock 1962). When voters have no uncertainty about their preferred alternative, using unanimity indeed guarantees that reforms will only be adopted when they satisfy the Pareto criterion. However, when voters are uncertain about which alternative is best, unanimity may perform poorly because it features poor information aggregation properties (Feddersen and Pesendorfer 1998). Obviously, nonunanimous rules (e.g. majority rule) are not panacea either, since they may fail to protect the minority from the tyranny of the majority. There is therefore a potential tension between protecting the minority and aggregating information.

In this paper, we analyze a simple voting system that essentially resolves this tension: majority rule with veto power (henceforth, we call this system Veto). Under Veto, agents have three possible actions (votes): yes, no, and veto. The reform is adopted if (i) a majority of agents vote yes, and (ii) no agent uses her veto. In all other cases, the status quo remains. We show that Veto combines the best of two worlds: it protects (even the smallest) minorities as well as the unanimity rule and it aggregates information at least as well as the majority rule. ${ }^{1}$

There are two rationales for our focus on the Pareto criterion. First, there are many real-world examples where satisfying the Pareto criterion is a stated objective or an underlying requirement. For instance, this is arguably the case for most institutions granting veto power to their members (e.g. EU, NATO, UN, American juries, and partnerships in professional service firms; see Romme 2004 for other examples). ${ }^{2}$ Second, this criterion is

\footnotetext{
${ }^{1}$ An alternative would be for the voting body to change its voting system depending on the kind of issue at hand: Unanimity when information is perfect and agents have private values, Majority when information is imperfect and agents have common values. The problem is that this requires the use of a complementary mechanism to decide which system to use. One might think that Veto is equivalent to using Unanimity to decide whether to use Unanimity or Majority. However, our results make clear that this is not the case.

${ }^{2}$ An oft-cited reason for a group to grant veto power to its members is to ensure their participation in the first place (see e.g. Wessel and Bottner 2013). Other reasons are that the group's decision requires the full support of its members (or a key subset of it) to be implemented (see e.g. Winter 1996, Wessel and Bottner 2013), and that it minimizes type-I mistakes (see e.g. the discussion in Feddersen and Pesendorfer 1998).
} 
at the heart of the Pareto efficiency concept which is ubiquitous in economics. A voting system that implements the Pareto criterion would potentially be very useful to correct an allocation which is not Pareto efficient (e.g. in the case of a market failure).

Agents of groups seeking Pareto improvements may be uncertain whether it is in their best interest to adopt the reform or keep the status quo. It may then be crucial that the voting system, on top of protecting even the smallest minority, aggregates the information dispersed among members. A striking example is that of the European Council when presiding over the Common Foreign and Security Policy. Common decisions on these matters can obviously hurt the private interests of a Member State. However, Member States may also have common objectives (e.g. facilitate the resolution of a violent conflict outside the $\mathrm{EU})^{3}$ but different information about the available alternatives (e.g. stemming from their respective regional expertise and/or intelligence agencies). For other examples see, e.g., Osborne and Turner (2009) and Bouton and Castanheira (2012).

To demonstrate the appealing properties of Veto, we compare it to the unanimity rule (Unanimity), and a simple majority rule (Majority) in a setup that captures the potential tension between minority protection and information aggregation. ${ }^{4}$ In particular, we generalize Feddersen and Pesendorfer (1998)'s model of group decision with private information and strategic voting to allow for (i) biases in the information structure (i.e. the relative precision of the signals), and (ii) the coexistence of common value and private value agents.

The main results are as follows. First, we characterize the set of equilibria under Veto and argue that only one equilibrium is relevant. The agents' behavior under Veto is intuitive and can be roughly described as a mix between what voters would do under Majority and what they would do under Unanimity. It has three distinctive features: (i) private value agents always use their veto power, (ii) common value agents behave as if there were no private value agents, and (iii) common value agents use their veto power

\footnotetext{
${ }^{3}$ More generally, "The Union's action on the international scene shall be guided by the principles which have inspired its own creation, development and enlargement (...): democracy, the rule of law, the universality and indivisibility of human rights and fundamental freedoms, respect for human dignity, the principles of equality and solidarity, and respect for the principles of the United Nations Charter and international law." (Art. 21 TEU 2012).

${ }^{4}$ Under Unanimity, agents have two possible actions (votes): yes, and veto. The reform is adopted if and only if no agent uses her veto (this is the same definition as in Feddersen and Pesendorfer 1998). Under Majority, agents have two possible actions (votes): yes, and no. The reform is adopted iff a majority of agents vote yes.
} 
only if the information structure is strongly biased.

Second, we use the concept of Full Information Pareto dominance to compare Veto with Majority and Unanimity. This concept, which is a direct application of the usual benchmark in the Condorcet Jury Theorem literature, relies on the ex ante probability of implementing the full information Pareto criterion. ${ }^{5}$ We show that, for any parameter values, Veto dominates both Unanimity and Majority and strictly dominates at least one of the two. This therefore implies the strict superiority of Veto at the "constitution writing stage" when the values of the parameters are still unknown.

Third, we focus on the comparison between Veto and Unanimity, which is the relevant comparison when the protection of the smallest minority is an essential feature of the system (it is arguably the case for the many real-world voting bodies using Unanimity ${ }^{6}$ ). Using the concept of Interim Pareto dominance (Holmstrom and Myerson 1983), we show that, even once types are realized, no agent can be worse off under Veto than Unanimity, and (except in some cases of lesser relevance) some agents are strictly better off. This implies that for any weighted utilitarian social welfare function, social welfare is weakly higher under Veto than under Unanimity. This generalizes the constitution-writing stage result above and leaves little ambiguity on the dominance of Veto over Unanimity. Furthermore, Interim Pareto dominance also implies that it would be a Pareto improvement in itself to adopt Veto in voting bodies that currently use Unanimity. This suggests that the adoption of Veto by those voting bodies should be relatively easy.

To conclude, we will argue that our results shed a new light on the evolution of voting rules in EU institutions since the Treaty of Maastricht, e.g. the introduction of "constructive abstention".

For the class of problems we study, a voting system can be considered optimal if it implements with probability one the decision that would be taken by a benevolent dictator who observes the type profile (but not the state) and applies the Pareto criterion. Although Veto clearly outperforms Majority and Unanimity, it is not always optimal in that sense. We identify an optimal system, but we see several good reasons to focus on Veto. First,

\footnotetext{
${ }^{5}$ The ex ante stage refers to the point in time at which agents know the parameters of the game (i.e the expected distribution of preferences and the potential biases in the relative precision of the signals), but do not know their type (i.e. their preferences and their signal). By contrast, the interim stage refers to the point in time at which agents also know their type.

${ }^{6}$ See the conclusion for examples.
} 
there is a broad range of parameter values for which Veto is optimal. Second, numerical examples suggest that, when Veto is not optimal, it still performs very well. Third, Veto is simple enough to have real life applications. ${ }^{7}$ Finally, Veto is cost-effective in the sense that it only requires one round of voting. By contrast, we conjecture that any system that is optimal for all parameter values requires multiple rounds of voting and/or relatively complex rules and ballot structures.

Our paper is related to four strands of the literature: first, the literature on the link between the unanimity rule and the Pareto criterion (e.g. Wicksell 1896; Buchanan and Tullock 1962; Guttman 1998; Chen and Ordeshook 1998; Sobel and Holcombe 1999; Dougherty and Edward 2005). Second, the literature studying aggregation of information in two-alternative decisions with strategic voters (see e.g. Austen-Smith and Banks 1996; Feddersen and Pesendorfer 1996, 1997, 1998; Myerson 1998; McLennan 1998; Chwe 1999; Guarnaschelli et al. 2000; Ali et al. 2008; Van Weelden 2008; Battaglini et al. 2008, 2010; Goeree and Yariv 2010; McMurray 2013; Bhattacharya et al. 2012). Third, the literature comparing electoral systems with either two alternatives (e.g. Feddersen and Pesendorfer 1998; Guarnaschelli et al. 2000; Coughlan 2001; Austen-Smith and Feddersen 2006; Bond and Eraslan 2009; Bouton et al. 2014) or multiple alternatives (e.g. Myerson and Weber 1993; Myerson 2000, 2002; Bouton and Castanheira 2012; Bouton et al. 2012; Bouton 2013). Fourth, the literature on veto power within institutions ( e.g. Cameron 2000; Woods 2000; Groseclose and McCarty 2001; Tsebelis 2002; Blum 2005; Maggi and Morelli 2006; Nunnari 2012).

\section{The Model}

A group of $n \geq 3$ agents (with $n$ odd) ${ }^{8}$ must choose between two possible alternatives, the status quo $B$ and the reform $R$.

Information structure. There are two possible states of nature: $\omega \in\left\{\omega_{B}, \omega_{R}\right\}$, which materialize with equal probability. The actual state of nature is not observable before the

\footnotetext{
${ }^{7}$ The argument is two fold: first, Veto is not too far from currently used voting systems (e.g. it requires a slight extension of action sets available under Majority or Unanimity and/or a change in approval quorum) so adjustment costs both in terms of infrastructures and in terms of voters' cognitive efforts should be low. Second, the equilibrium behavior of voters is barely more sophisticated than under Majority or Unanimity. Voters should thus easily understand how to behave in the "right" way under Veto.

${ }^{8}$ That $n$ is odd only simplifies the exposition.
} 
vote, but each agent has private information about it. In particular, before voting, each agent privately observes an imperfectly informative signal. ${ }^{9}$ Conditional on the state, the signals are independently drawn. There are two possible signals: $s_{B}$ and $s_{R}$, where the probability for an agent to observe signal $s_{R}$ is higher in state $\omega_{R}$ than in state $\omega_{B}$, and conversely for $s_{B}$ :

$$
\operatorname{Pr}\left(s_{R} \mid \omega_{R}\right)>\operatorname{Pr}\left(s_{R} \mid \omega_{B}\right)>0 \text { and } \operatorname{Pr}\left(s_{B} \mid \omega_{B}\right)>\operatorname{Pr}\left(s_{B} \mid \omega_{R}\right)>0
$$

This assumption is without loss of generality. In particular, it allows for cases in which observing a given signal is more likely than the other in both states, e.g. $\operatorname{Pr}\left(s_{B} \mid \omega_{R}\right)>$ $\operatorname{Pr}\left(s_{R} \mid \omega_{R}\right)$.

Types and preferences. Agents may have different preferences over outcomes. To simplify the notation, we assume that the type of an agent, denoted by $z \in\left\{z_{R}, z_{B}, z_{P}\right\}$, captures both the signal she received and her preferences.

Agents of types $z_{R}$ and $z_{B}$ have the same state-contingent preferences: conditional on the state of nature, their preferred group decision is the same, that is $B$ in state $\omega_{B}$ and $R$ in state $\omega_{R}$. Denoting by $u_{z}(D, \omega)$ the (von Neumann-Morgenstern) utility function of an agent of type $z$ when the group decision is $D$ and the state of nature is $\omega$, we have:

$$
\begin{aligned}
u_{z_{B}}(D, \omega)=u_{z_{R}}(D, \omega) & =0 \text { if }(D, \omega)=\left(B, \omega_{B}\right) \text { or }\left(R, \omega_{R}\right) \\
& =-1 \text { if }(D, \omega)=\left(R, \omega_{B}\right) \text { or }\left(B, \omega_{R}\right),
\end{aligned}
$$

The only difference between agents of types $z_{B}$ and $z_{R}$ is that they have different interim beliefs about the state of nature, for they have received a signal $s_{B}$ and $s_{R}$, respectively.

\footnotetext{
${ }^{9}$ Endogenizing information acquisition is out of the scope of this paper (for more details on this issue, see e.g. Persico 2004, Martinelli 2006, 2007, and Gerardi and Yariv 2008). However, note that Unanimity does not necessarily promote information aquisition more than other rules. Assuming exogenous private information is therefore not a way to stack the deck in favor of Veto. Indeed, Persico (2004) shows that Unanimity is optimal with respect to information acquisition only if information available to each agent is sufficiently precise. There are reasons to believe that Veto would feature similar properties in those cases, since, as we show below, when information is "very" precise, the voting behavior of agents under Veto mimicks the one under Unanimity. For the comparison of Veto and Majority, it is true that the veto power granted to every agent (in particular to private value agents) should negatively affect incentives to acquire information under Veto. Yet, as we show below, for some level of accuracy, other types of agents use their veto power with positive probability. In those cases, the incentives to acquire information should be higher under Veto than Majority because the likelihood of being pivotal is higher under the former than the latter. The overall effect of granting veto power to every agent is thus unclear.
} 
We refer to these agents as having common values. The problem these agents face at the time of the vote is essentially one of information aggregation.

In contrast, agents of type $z_{P}$ prefer the status quo $B$ in both states of nature:

$$
\begin{aligned}
& u_{z_{P}}(B, \omega)=0 \forall \omega \\
& u_{z_{P}}(R, \omega)=-1 \forall \omega .
\end{aligned}
$$

We refer to these agents as having private values. ${ }^{10}$ Given these preferences, such agents can be made worse off by a majority decision.

By a slight abuse of notation, we denote by $z_{i}$ the type of agent $i$. To capture the potential tension between information aggregation and minority protection, we assume that agents are ex ante identical and face a probability $\beta \in[0,1)$ of being assigned the private value type (i.e. $z_{i}=z_{P}$ ), and a probability $1-\beta$ of being assigned a common value type (i.e. $\left.z_{i} \in\left\{z_{B}, z_{R}\right\}\right) .{ }^{11}$ The realized type profile is denoted by $\mathbf{z} \in\left\{z_{R}, z_{B}, z_{P}\right\}^{n}$. For simplicity, the probability $\beta$ is assumed to be identical for all agents, and independent from the state of nature $\omega$. None of the main results depend on this simplification.

Voting systems. We consider group decisions under three voting systems: Veto $(V)$, Unanimity $(U)$, and Majority $(M)$. Each agent has to choose an action, which can be interpreted as a vote. A voting system $\Psi \in\{U, M, V\}$ is defined as a set of possible actions $A^{\Psi}$ and a decision rule $d^{\Psi}$ mapping agents' actions into a group decision: $d^{\Psi}$ : $\left\{a \in A^{\Psi}\right\}^{n} \rightarrow\{B, R\}$. We denote by $X_{a}$ the total number of agents playing action $a$. Agents do not communicate (or it is too costly for them to do so) before making their decision. ${ }^{12,13}$

\footnotetext{
${ }^{10}$ Our setup aims at capturing the tension between minority protection and information aggregation in the simplest possible way. For the sake of symmetry, we could have considered pro-reform private value agents. However, this would not have added much to the analysis since we focus on groups that want to reform the status quo only if it is Pareto improving.

${ }^{11}$ The case where $\beta=1$ is uninteresting as all agents would always prefer the status quo and there is neither a need to aggregate information nor a potential divergence in agents interest.

${ }^{12}$ The literature suggests that, for nonunanimous voting rules, communication helps committees make better decisions when an underlying consensus exists in the committee or, if there is no such consensus, when some conditions on the structure of uncertainty about agents' preferences are satisfied (e.g. Coughlan 2000, and Austen-Smith and Feddersen 2006). The literature also shows that unanimity features bad properties with respect to information sharing, even when information sharing is possible under nonunanimous rules (e.g. Gerardi and Yariv 2007, and Austen-Smith and Feddersen 2006). For an insightful discussion of this topic see Persico (2004, pp. 170-171).

${ }^{13}$ In our setup, perhaps surprisingly, full information sharing is possible under Unanimity and Veto, but not under Majority. Information sharing under Unanimity and Veto is possible because agents have a veto
} 
Definition 1 Voting system "Veto" is defined by: $V \equiv\left\{A^{V}, d^{V}\right\}$, where:

$$
\begin{aligned}
A^{V} & =\{r, b, v\} \\
d^{V} & =\left\{\begin{array}{cc}
R & \text { if } X_{v}=0 \text { and } X_{r}>X_{b} \\
B & \text { otherwise. }
\end{array}\right.
\end{aligned}
$$

Equivalently, looking at the complement gives: the group decision is $B$ if either $X_{v} \geq$ 1 or $X_{r} \leq X_{b}$. One can interpret the actions as follows. First, since a single $v$ suffices to enforce the status quo $B$, it can be interpreted as a veto exercised against the reform $R$. Second, $b$ can be either interpreted as a vote for the status quo $B$, or against the reform $R$. Conversely, $r$ can be either interpreted as a vote for the reform $R$, or against the status quo $B$. We will often use following terminology: action $r$ is a vote for $R$, action $b$ is a vote for $B$, and action $v$ is a veto (against $R$ ).

It is convenient to define $U$ and $M$ using the same decision rule $\left(d^{V}\right)$. This helps to highlight the differences and similarities between $M, U$ and $V$.

Definition 2 Voting system "Unanimity" is defined by: $U \equiv\left\{A^{U}, d^{V}\right\}$, where:

$$
\begin{aligned}
& A^{U}=\{r, v\} \subset A^{V} \\
& d^{U}=d^{V}
\end{aligned}
$$

with $X_{b}$ necessarily equal to 0 .

Under $U, r$ can still be considered as a vote for $R$. However, the only action that weigh against $R$ is $v$. It can either be interpreted as a vote for $B$ where unanimity is required to implement $R$, or as a veto.

Definition 3 Voting system "Majority" is defined by: $M \equiv\left\{A^{M}, d^{V}\right\}$, where:

$$
\begin{aligned}
& A^{M}=\{b, r\} \subset A^{V} \\
& d^{M}=d^{V}
\end{aligned}
$$

power: private value agents do not have an incentive to lie about their type because they can use their veto at the voting stage, and hence ensure that the status quo remains. Under Majority, private value agents have an incentive to lie about their type since they cannot unilaterally decide that the status quo remains. In particular, they have an incentive to claim that they received signal $s_{B}$. 
with $X_{v}$ necessarily equal to 0 .

Under $M, r$ is naturally considered as a vote for $R$, and $b$ as a vote for $B$. All votes have the same weights, and no veto is possible.

Three remarks are in order.

Remark 1 Veto can also be interpreted as a modified version of Unanimity, where abstention is allowed and an approval quorum is required for the reform to be adopted. To see that, just relabel the three possible actions under Veto "yes", "abstention", and "no" respectively, and note that for the group decision to be $R$, one requires i) a unanimity of "yes" among agents who do not abstain, and ii) more "yes" than abstention.

Remark 2 A modified version of Unanimity, where abstention is allowed, but no quorum is required, is strategically equivalent to Unanimity. Indeed, in that case, adopting the reform only requires that there is no vote against it among agents that do not abstain, and voting yes or abstaining is therefore strategically equivalent. This voting system is often referred to as "Consensus".

Remark 3 For the sake of simplicity, we consider a threshold $q=\frac{n+1}{2}$ under Veto and Majority, i.e. the reform is implemented only if there are $q$ or more votes for $r$. We would obtain similar welfare results considering, for instance, any (supermajority) threshold $q \in$ $\left[\frac{n+1}{2}, n-1\right]$.

Strategy and equilibrium concept. Formally, we define an agent strategy under voting system $\Psi$ as a function $\sigma^{\Psi}:\left\{z_{R}, z_{B}, z_{P}\right\} \rightarrow \triangle\left(A^{\Psi}\right)$. In particular, $\sigma_{a}^{\Psi}(z)$ denotes the probability with which an agent of type $z$ votes $a$ under system $\Psi$. Following Feddersen and Pesendorfer (1998), we focus on responsive symmetric Bayesian Nash equilibria. A responsive profile is such that (i) at least some common value agents play action $r$ with positive probability, and (ii) not all of them play $r$ with probability 1, i.e. $\sigma_{r}^{\Psi}\left(z_{R}\right)+$ $\sigma_{r}^{\Psi}\left(z_{B}\right) \in(0,2)$. This ensures that, in equilibrium, some pivot probabilities are strictly positive, i.e. agents affect the outcome of the vote with positive probability. We focus on 
responsive equilibria to exclude equilibria in which all common value agents vote the same way independent of their type. For instance, under $U$ and $V$, if all common value agents vote $v$, then no agent can influence the group decision by deviating. Hence, this is an equilibrium. Ruling out weakly dominated strategies does not eliminate such equilibria.

Our focus on responsive equilibria implies that agents of type $z_{P}$ have a strictly dominant strategy. Indeed, given that the reform $R$ is implemented with positive probability for any responsive strategy profile, private value agents always strictly prefer to vote against $R$. Hence, $z_{P}$-agents use their veto under $V$ and $U$, and vote $b$ under $M$ :

$$
\sigma_{v}^{V}\left(z_{P}\right)=\sigma_{v}^{U}\left(z_{P}\right)=\sigma_{b}^{M}\left(z_{P}\right)=1
$$

\section{Equilibrium Analysis}

In this section, we characterize the set of responsive symmetric equilibria under Veto and argue that only one equilibrium is relevant. The characterization of the unique responsive symmetric equilibrium under Unanimity being a straightforward extension of Feddersen and Pesendorfer (1998), we relegate the details to the Supplementary Appendix. Finally, we do not characterize the set of responsive symmetric equilibria under Majority because our welfare analysis only requires that one such equilibrium exists (which is trivially guaranteed in our setting).

We organize the analysis as follows: first, in Subsection 3.1, we show that the behavior of common value agents is independent of $\beta$, i.e. they always behave as in a "pure common value game" $(\beta=0)$. Second, we define the pivotal events in this common value game, compute their probabilities, and derive the expected payoffs of the different actions (Subsection 3.2.1). Finally, we characterize the set of equilibria (Subsection 3.2.2). For the sake of readability, we abstract from the superscript $\Psi$ in this section since it is always $V$.

\subsection{The Common Value Equivalence}

The following proposition shows that any equilibrium under $V$ must feature two specific characteristics: 
Proposition 1 For any value of $\beta \in[0,1), \sigma^{*}$ is a responsive symmetric equilibrium under Veto if and only if:

i) private value agents veto the reform, ii) $\sigma^{*}$ is a responsive symmetric equilibrium of the game corresponding to $\beta=0$.

Proof. See Appendix A1.

The intuition behind this result is as follows: given that $z_{P}$-agents always use their veto power, each common value agent realizes that her behavior may affect the group decision only if all other agents have common values. Hence, they condition their behavior on that event. Therefore, the strategy of common value agents must always be an equilibrium of the game in which all agents have common values with probability 1 (i.e. $\beta=0$ ).

This proposition greatly simplifies the characterization of an equilibrium under Veto since it allows us to focus on the pure common value game in which $\beta=0$.

\subsection{The Common Value Game}

In the pure common value game, $\beta=0$. Hence, there are $n$ common value agents, i.e. $z \in\left\{z_{B}, z_{R}\right\}$ for all agents. We will often refer to $z_{B}$ and $z_{R}$ as signals instead of types.

Agents' behavior depend on pivotal events: situations in which their vote changes the final outcome towards a specific group decision. In other words, an agent is pivotal if the group decision would be different without her vote. Whether a vote is pivotal therefore depends on the decision rule and on all other agents' behavior. Agents' behavior, in turn, depends on their strategies and on the signal they receive. Thus, for any strategy profile, it is possible to compute the probability of each pivotal event.

At this point, it is useful to introduce two new objects. First, $x_{a}$ denotes, from the perspective of a given agent, the number of other agents playing action $a$. Second, $\tau_{a}^{\omega}(\sigma)$ denotes the state-contingent probability that an agent votes $a$ in state $\omega$ for a given strategy profile $\sigma$. It is defined as follows:

$$
\tau_{a}^{\omega}(\sigma) \equiv \sum_{z \in\left\{z_{B}, z_{R}\right\}} \sigma_{a}(z) \operatorname{Pr}(z \mid \omega)
$$

where $\operatorname{Pr}\left(z_{R} \mid \omega\right)=\operatorname{Pr}\left(s_{R} \mid \omega\right)$ and $\operatorname{Pr}\left(z_{B} \mid \omega\right)=\operatorname{Pr}\left(s_{B} \mid \omega\right) \forall \omega$ since $\beta=0$. 


\subsubsection{Pivot Probabilities and Payoffs}

Under Veto, there are two pivotal events. First, a $r$-vote is pivotal when, without that vote, $R$ is lacking just one vote to be adopted (i.e. $x_{b}=x_{r}$ ) and nobody cast a $v$-vote. We denote that pivotal event in state $\omega$ by $p_{i} v_{R}^{\omega}$. Second, a $v$-vote is pivotal when the number of $r$-votes among other agents is strictly larger than the number of $b$-votes (i.e. $x_{r}>x_{b}$ ) and nobody else casts a $v$-vote. We denote that pivotal event in state $\omega$ by $p i v_{B}^{\omega}$. Importantly, a $b$-ballot is never pivotal under Veto. Indeed, this would require that, without that vote, $R$ wins (i.e. $\left.x_{r}>x_{b}\right)$, and, with that vote, $B$ wins $\left(X_{r} \leq X_{b}\right)$; an impossibility since $n$ is odd.

For the sake of readability, our notation does not reflect the fact that the probability of pivotal events depend on the strategies through the expected vote shares, i.e. we henceforth omit $\sigma$ from the notation.

For $\operatorname{piv}_{R}^{\omega}$, we have

$$
\operatorname{Pr}\left(p i v_{R}^{\omega}\right)=\left(\begin{array}{c}
n-1 \\
\frac{n-1}{2}
\end{array}\right)\left(\tau_{b}^{\omega}\right)^{\frac{n-1}{2}}\left(\tau_{r}^{\omega}\right)^{\frac{n-1}{2}}
$$

Similarly, for piv $_{B}^{\omega}$ we have:

$$
\operatorname{Pr}\left(p_{i v}^{\omega}\right)=\sum_{j=\frac{n+1}{2}}^{n-1}\left(\begin{array}{c}
n-1 \\
j
\end{array}\right)\left(\tau_{r}^{\omega}\right)^{j}\left(\tau_{b}^{\omega}\right)^{n-1-j}
$$

Using these pivot probabilities, we can compute the expected payoff of the different actions for a common value agent of type $z \in\left\{z_{B}, z_{R}\right\}$. To do this, it is useful to define common value agents' interim beliefs about the state of nature:

$$
\begin{aligned}
\operatorname{Pr}\left(\omega_{B} \mid z\right) & =\frac{\operatorname{Pr}\left(z \mid \omega_{B}\right)}{\operatorname{Pr}\left(z \mid \omega_{B}\right)+\operatorname{Pr}\left(z \mid \omega_{R}\right)}, \text { and } \\
\operatorname{Pr}\left(\omega_{R} \mid z\right) & =\frac{\operatorname{Pr}\left(z \mid \omega_{R}\right)}{\operatorname{Pr}\left(z \mid \omega_{R}\right)+\operatorname{Pr}\left(z \mid \omega_{B}\right)} .
\end{aligned}
$$

Therefore, from (1), we have that the expected payoff of an $r$-vote for a common value agent who received signal $z$ is

$$
G(r \mid z)=\operatorname{Pr}\left(\omega_{R} \mid z\right) \operatorname{Pr}\left(p_{i} v_{R}^{\omega_{R}}\right)-\operatorname{Pr}\left(\omega_{B} \mid z\right) \operatorname{Pr}\left(\text { piv }_{R}^{\omega_{B}}\right)
$$


the expected payoff of a $v$-vote for a common value agent who received signal $z$ is

$$
G(v \mid z)=\operatorname{Pr}\left(\omega_{B} \mid z\right) \operatorname{Pr}\left(p i v_{B}^{\omega_{B}}\right)-\operatorname{Pr}\left(\omega_{R} \mid z\right) \operatorname{Pr}\left(p i v_{B}^{\omega_{R}}\right)
$$

and the expected payoff of a $b$-vote for a common value agent who received signal $z$ is

$$
G(b \mid z)=0
$$

\subsubsection{Voting Behavior}

To organize the discussion of the equilibrium behavior of common value agents under Veto, it is useful to partition the parameter space. This is because equilibrium strategies are non-trivially affected by the relative precision of the signals. We denote the precision of a

signal $z_{B}$ by $\phi_{B} \equiv \frac{\operatorname{Pr}\left(z_{B} \mid \omega_{B}\right)}{\operatorname{Pr}\left(z_{B} \mid \omega_{R}\right)}$ and that of signal $z_{R}$ by $\phi_{R} \equiv \frac{\operatorname{Pr}\left(z_{R} \mid \omega_{R}\right)}{\operatorname{Pr}\left(z_{R} \mid \omega_{B}\right)}$. For any $n$ and $\phi_{R}$, we have identified four thresholds $\phi_{1}, \phi_{2}, \phi_{3}, \phi_{4}$ for $\phi_{B}$, at which the set of actions played with strictly positive probability in equilibrium changes (see the proof of Proposition 2 in Appendix A1 for their formal definition, and the Supplementary Appendix for a proof that $\left.1<\phi_{1}<\phi_{2} \leq \phi_{3} \leq \phi_{4}<\infty\right)$.

We now state our main result about the behavior of common value agents under Veto:

Proposition 2 Under Veto, for each $\phi_{R}, \phi_{B}, n$, there exist unique $\eta^{*}, \epsilon^{*}, \gamma^{*}, \delta^{*}$ satisfying

$\begin{array}{cccccc}\phi_{B} & <\phi_{1} & \in\left[\phi_{1}, \phi_{2}\right] & \in\left(\phi_{2}, \phi_{3}\right] & \in\left(\phi_{3}, \phi_{4}\right) & \geq \phi_{4} \\ \eta^{*} & {[0,1)} & 0 & 0 & 0 & 0 \\ \epsilon^{*} & 0 & 0 & (0,1) & 0 & 0 \\ \gamma^{*} & 0 & 0 & 0 & \left(0,1-\delta^{*}\right) & 0 \\ \delta^{*} & 0 & 0 & 0 & (0,1) & 1\end{array}$

such that the following strategy profile is a responsive symmetric equilibrium:

$$
\begin{array}{ccc}
\sigma_{r}\left(z_{R}\right)=1-\epsilon^{*}-\gamma^{*} & \sigma_{b}\left(z_{R}\right)=\epsilon^{*}+\gamma^{*} & \sigma_{v}\left(z_{R}\right)=0 \\
\sigma_{r}\left(z_{B}\right)=\eta^{*} & \sigma_{b}\left(z_{B}\right)=1-\eta^{*}-\delta^{*} & \sigma_{v}\left(z_{B}\right)=\delta^{*}
\end{array}
$$


Proof. See Appendix A1.

Figure 1 illustrates Proposition 2 in the space $\left(\phi_{R}, \phi_{B}\right)$ for $n=5$. In short, the voting behavior under Veto is as follows. For $\phi_{B} \in\left[1, \phi_{3}\right]$, the behavior of common value agents under Veto is the same as it would be under Majority, and for $\phi_{B} \in\left[\phi_{4}, \infty\right)$ it is the same as it would be under Unanimity. For $\phi_{B} \in\left(\phi_{3}, \phi_{4}\right)$, all actions are played with positive probability. Therefore, the behavior under Veto is necessarily different than under Majority or Unanimity. We now detail the mechanisms behind the behavior of common value agents for the different values of $\phi_{B}$.

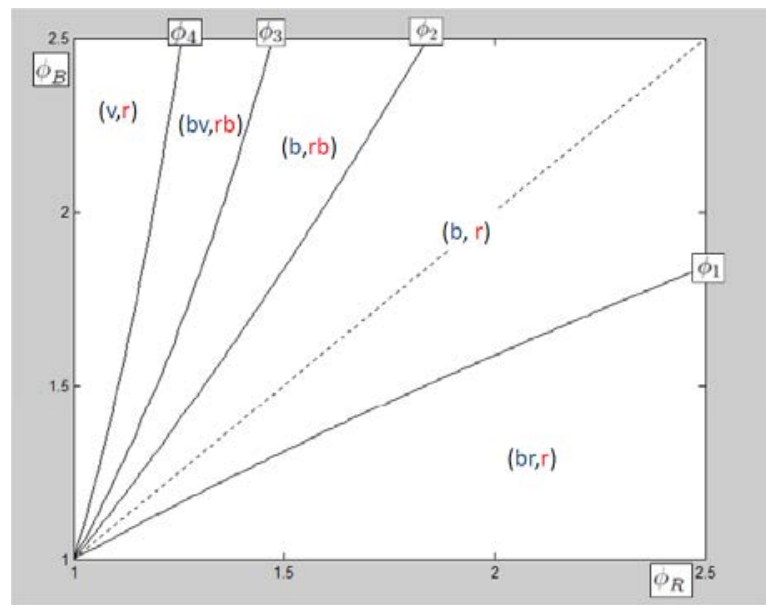

Figure 1: Equilibrium strategies under Veto for $n=5$. The colored letters in parentheses refer to the strategy of the common-value agents. The first element of each couple (in blue) refers to the strategy of $z_{B}$-agents. The second element of each couple (in red) refers to the strategy of $z_{R}$-agents. Pure strategies are denoted by single letters, mixed strategies are denoted by two letters.

When $\phi_{B} \in\left[\phi_{1}, \phi_{2}\right]$, the precision of the two signals is not too different. The equilibrium is then such that $\sigma_{r}\left(z_{R}\right)=1=\sigma_{b}\left(z_{B}\right)$ : common value agents vote their signal and do not use their veto. The intuition is the following. An $r$-ballot is pivotal if $R$ and $B$ receive the same number of votes in the group of other agents. Given the strategy under consideration, this requires that in the group of other agents, there are $\frac{n-1}{2}$ signals $z_{B}$ and $\frac{n-1}{2}$ signals $z_{R}$. Adding one's own signal to this count means a lead of one signal in favor of one of the two states. The condition $\phi_{B} \in\left[\phi_{1}, \phi_{2}\right]$ ensures that, in such a case, the posterior beliefs of the agent under consideration are in line with her signal. In other

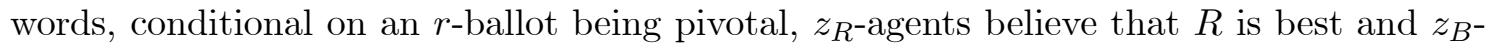


agents believe that $B$ is best. The implication in terms of voting behavior is obvious for

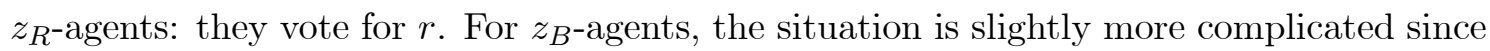
there are two options to vote against $R$ : voting either $b$ or $v$. A $b$-ballot has an expected payoff of zero, whereas a $v$-ballot has a negative expected payoff. This is so because a $v$-ballot changes the outcome when there are more $r$ votes than $b$ votes. Given the strategy under consideration and the relative precision of the signals, this is much more likely to happen in state $\omega_{R}$ than in state $\omega_{B}$.

When $\phi_{B} \in\left[1, \phi_{1}\right)$, signal $z_{R}$ is more precise than signal $z_{B}$. In that case, $z_{B}$-agents prefer to overlook their signal and vote $r$ with positive probability. Doing so, they "compensate" for the bias in the information structure. The reason is that, because the signal is imprecise, the probability of making a mistake in state $\omega_{R}$ is too high. This is exactly the same behavior as under Majority.

The case with $\phi_{B} \in\left(\phi_{2}, \phi_{3}\right]$ resembles the one with $\phi_{B} \in\left[1, \phi_{1}\right)$, but the difference in signal precision is in favor of $z_{B}$. As a result of the difference in signal precision, $z_{R}$-agents prefer to overlook their signal and vote against $R$ with positive probability. Again, this is similar as under Majority. Yet, under Veto agents have two ways to vote against $R$ : voting $b$ or $v$. The appeal of $v$ depends positively on the precision of the $z_{B}$ signal, and negatively on the relative probability of being pivotal in favor of $B$ in states $\omega_{R}$ and $\omega_{B}$. Therefore, $\phi_{3}$ requires that, for a given precision of the $z_{B}$ signal, the expected lead of $R$ in state $\omega_{R}$ is large enough and/or the lead of $B$ in state $\omega_{B}$ small enough.

When $\phi_{B} \in\left(\phi_{3}, \phi_{4}\right)$, the situation resembles the situation for $\phi_{B} \in\left(\phi_{2}, \phi_{3}\right]$. The difference is that $z_{B}$-agents want to use their veto power with positive probability. As just explained, this is so because, for the strategy profile when $\phi_{B} \in\left(\phi_{2}, \phi_{3}\right]$, the expected lead of $R$ in state $\omega_{R}$ is too large in comparison to the expected lead of $B$ in state $\omega_{B}$. In

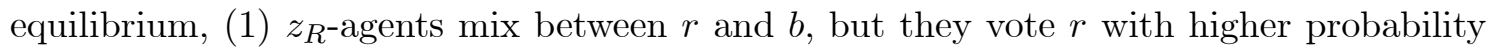
than for $\phi_{B} \leq \phi_{3}$, and (2) $z_{B}$ agents mix between $b$ and $v$. The intuition comes in two steps. First, the positive probability of a veto by $z_{B}$ agents makes a vote $r$ more appealing (i.e. $\operatorname{Pr}\left(p i v_{R}^{\omega_{R}}\right) / \operatorname{Pr}\left(\right.$ piv $\left.\omega_{R}^{\omega_{B}}\right)$ goes up since there are more $z_{B}$ agents in state $\left.\omega_{B}\right)$. Second, the relatively higher vote shares of $R$ in state $\omega_{R}$ makes a $v$ vote less appealing (i.e. $\operatorname{Pr}\left(\right.$ piv $\left.\omega_{B}^{\omega_{R}}\right)$ goes up and $\operatorname{Pr}\left(\right.$ piv $\left._{B}^{\omega_{B}}\right)$ goes down $)$.

When $\phi_{B} \in\left[\phi_{4}, \infty\right)$, common value agents behave as they would under Unanimity. The 
$z_{B}$ signal is so precise (relatively) that one $z_{B}$ signal is sufficient information to conclude that $B$ is better than $R$ (even if all other signals are $z_{R}$ signals). Therefore, $z_{B}$-agents prefer to cast a $v$-vote. For $z_{R}$ agents, the situation is different. Given the strategy under consideration, conditional on being pivotal, all other agents must have received a $z_{R}$ signals. Obviously, if there are only $z_{R}$ signals, any agent must believe that state $\omega_{R}$ is more likely than state $\omega_{B}$. She thus prefers to cast a $r$-vote.

Multiplicity and Stability We show here that when $\phi_{B}<\phi_{4}$, there exists another symmetric responsive equilibrium, which corresponds to the unique equilibrium under Unanimity (in the Supplementary Appendix, we show that this is the only other equilibrium). However, such an equilibrium is not robust under Veto, for it is both unstable and Pareto Dominated. We therefore see it as of little relevance. In a companion paper, we present the results of an experimental study that strongly supports this view (See Bouton et al., 2014).

Proposition 3 For each $\phi_{R}, \phi_{B}$, n, the following strategy profile is a responsive symmetric equilibrium under Veto:

$$
\begin{array}{ccc}
\sigma_{r}\left(z_{R}\right)=1 & \sigma_{b}\left(z_{R}\right)=0 & \sigma_{v}\left(z_{R}\right)=0 \\
\sigma_{r}\left(z_{B}\right)=\alpha^{*} & \sigma_{b}\left(z_{B}\right)=0 & \sigma_{v}\left(z_{B}\right)=1-\alpha^{*}
\end{array}
$$

where $\alpha^{*}=\frac{\left(\phi_{B}-1\right)\left(\phi_{R}-\left(\phi_{B}\right)^{\frac{1}{n-1}}\right)}{\left(\phi_{R}-1\right)\left(\left(\phi_{B}\right)^{\frac{1}{n-1}} \phi_{B}-1\right)} \in[0,1)$.

This equilibrium corresponds to the unique equilibrium under Unanimity.

Proof. Straightforward extension of Feddersen and Pesendorfer (1998). See the Supplementary Appendix (section Unanimity) for a full characterization.

In fact, when $\phi_{B} \geq \phi_{4}$, this equilibrium is identical to that described in Proposition 2 (i.e. $\alpha^{*}=\eta^{*}$, and $1-\alpha^{*}=\delta^{*}$ ). Hence, in this region, there is a unique and identical equilibrium under Veto and Unanimity.

Under unanimity, $z_{B}$-agents realize they can only be pivotal if all other agents vote $r$. Given that this is more likely to happen in state $\omega_{R}$ (because $z_{R}$-agents always play $r$ ), $z_{B}$-agents only play $v$ with probability 1 if signal $s_{R}$ is relatively hyper-precise $\left(\phi_{B} \geq \phi_{4}\right)$. 
In all other cases, they play $r$ with positive probability, which results in a relatively high probability of errors of both types (Feddersen and Pesendorfer 1998).

To understand why it is also an equilibrium under Veto, recall that Unanimity corresponds to Veto with a smaller action set. That is, action $b$ is not available under Unanimity. Under Veto, when no other agent ever votes $b$, a $b$-vote is strategically equivalent to an $r$-vote (since there is a zero probability to have a majority of $b$, the reform will be adopted unless someone votes $v) .{ }^{14}$ So, the equilibrium under Unanimity must be an equilibrium under Veto.

However, the possibility to vote $b$ makes this equilibrium unstable. Imagine that $z_{B^{-}}$ agents tremble and play $b$ with very small but strictly positive probability $\theta$ (noting that in equilibrium, they are in fact indifferent between the three possible actions), while $z_{R^{-}}$ agents still play $r$ with probability 1 . Then, a best response for $z_{B}$-agents cannot involve playing both $r$ and $v$ with strictly positive probability, i.e. it cannot be "close" to the equilibrium strategy profile. In fact, as $\theta$ tends to 0 , the equilibrium of such a perturbed game tends to the equilibrium in Proposition 2.

The intuition is the following: in Proposition 3's equilibrium, agents are indifferent between $b$ and $r$ because one can never be pivotal in favor of $R$ (if no other voter has vetoed, then it must be that everyone else played $r$, and there already is a majority in favor of the reform). But, if $b$ is played with positive probability, even very small, it becomes possible that $X_{r}=\frac{n-1}{2}$. Since this is much more likely to happen in state $B$ than in state $R$, a $z_{B}$-agent strictly prefers to vote $b$ than $r$. Note that if agents $z_{B}$ do not play $r$, this decreases the gain for them to play $v$, and the equilibrium unravels. ${ }^{15}$

For these reasons, and because this equilibrium is Pareto dominated by that of Proposition 2, we will focus on the latter when comparing welfare across voting systems. Note that if agents were nevertheless to play the former, outcomes would simply be equivalent under Veto and Unanimity and welfare properties would be identical.

\footnotetext{
${ }^{14}$ In terms of information aggregation, playing $b$ at such an equilibrium could convey information, but such information would not be exploited in taking the group decision.

${ }^{15}$ Note that the equilibrium is however trembling hand perfect because it is possible to find a joint sequence of tremble for all agents that tends to 0 , and a corresponding sequence of equilibria that converges towards the equilibrium. However, it is easy to show that such sequences must have the unappealing feature that trembles make agents $z_{R}$ vote more for $b$ than agents $z_{B}$.
} 


\section{Welfare Analysis}

In this section, we assess the welfare properties of Veto in two steps. First, we highlight Veto's general desirable properties by comparing it to both Majority and Unanimity with a benchmark directly adapted from the Condorcet Jury Theorem literature. We use the concept of Full Information Pareto dominance, which is based on the ex ante probability of implementing the Pareto criterion. We show that Veto dominates both Unanimity and Majority and strictly dominates at least one of the two. This result holds for all possible values of $\phi_{B}, \phi_{R}, n$, and $\beta$, and which of Unanimity or Majority is strictly dominated depends on the parameter values. This therefore implies the strict superiority of Veto at the "constitution writing stage" where $\phi_{B}, \phi_{R}, n$, and $\beta$ are still unknown.

Second, we focus on the comparison between Veto and Unanimity, which is the relevant comparison when the protection of the smallest minority is an essential feature of the system (it is arguably the case for institutions currently using Unanimity). Using the concept of Interim Pareto dominance (Holmstrom and Myerson 1983), we show that, even once types are realized, no agent can be worse off under Veto than Unanimity, and (except in some cases of lesser relevance) some agents are strictly better off. This implies that for any weighted utilitarian social welfare function, social welfare is weakly higher under Veto than under Unanimity. This generalizes the constitution-writing-stage result above and leaves little ambiguity on the dominance of Veto over Unanimity in this context. Furthermore, Interim Pareto dominance also implies that it would be a Pareto improvement in itself to adopt Veto in voting bodies that currently use Unanimity. This suggests that the adoption of Veto by those voting bodies should be relatively easy.

\subsection{Full Information Pareto Dominance}

In the spirit of the Condorcet Jury Theorem literature (see e.g. Feddersen and Pesendorfer 1997), we define the Full Information Pareto decision (FIP decision) as the decision that satisfies the Pareto criterion when the type profile and state are known. ${ }^{16}$ Formally, it is

\footnotetext{
${ }^{16}$ This benchmark decision corresponds to the full information equivalence benchmark ubiquitous in the Condorcet Jury Theorem literature. It is the decision that would be taken by the group if they knew the state of nature and were using Unanimity or Veto.
} 
given by:

$$
D^{F I P C}(\mathbf{z}, \omega)= \begin{cases}R & \text { if } z_{i} \neq z_{P} \forall i, \text { and } \omega=\omega_{R} \\ B & \text { otherwise }\end{cases}
$$

The logic is straightforward. Private value agents (if any) are strictly worse off if $R$ is chosen. In the absence of such agents, the relevant question is whether the reform is in the best interest of common value agents. This is the case when the state is $\omega_{R}$.

To assess the welfare properties of the voting systems using the interim probability of taking this decision, we first need to define

$$
D\left(\sigma^{* \Psi}, \mathbf{z}\right):\left\{\sigma^{* \Psi}, \mathbf{z}\right\} \rightarrow \triangle(\{B, R\}),
$$

the group decision mapping implied by voting system $\Psi$, for the associated equilibrium strategy profile $\sigma^{* \Psi}$ when the realized type profile is $\mathbf{z}$. Given $\phi_{B}, \phi_{R}, n$ and $\beta$, the interim probability of taking the FIP Decision under voting system $\Psi$ is:

$$
\Pi\left(\Psi, \sigma^{* \Psi}, \mathbf{z}, \omega\right) \equiv \operatorname{Pr}_{\sigma}\left(D\left(\sigma^{* \Psi}, \mathbf{z}\right)=D^{F I P C}(\mathbf{z}, \omega)\right) \in[0,1] .
$$

Let us now define the concept of Full Information Pareto Dominance:

Definition 4 A voting system $\Psi$ Full Information Pareto Dominates (FIP Dominates) another voting system $\Psi^{\prime}$, for given $\phi_{B}, \phi_{R}, n$ and $\beta$, if there exists an equilibrium under

$\Psi$ such that $E_{\mathbf{z}, \omega}\left[\Pi\left(\Psi, \sigma^{* \Psi}, \mathbf{z}, \omega\right)\right] \geq E_{\mathbf{z}, \omega}\left[\Pi\left(\Psi^{\prime}, \sigma^{* \Psi^{\prime}}, \mathbf{z}, \omega\right)\right]$ for all $\sigma^{* \Psi^{\prime}}$, and $\Psi$ Strictly FIP Dominates $\Psi^{\prime}$ if this condition holds with strict inequality.

Our first welfare result is stated in the following theorem:

Theorem 1 For all $\phi_{B}, \phi_{R}, n$, and $\beta$, Veto FIP dominates both Majority and Unanimity. Unless $\beta=0$ and $\phi_{B} \leq \phi_{3}$, Veto Strictly FIP dominates Majority. Unless $\phi_{B} \geq \phi_{4}$, Veto Strictly FIP dominates Unanimity.

Proof. See Appendix A2.

To understand the first part of Theorem 1, first notice that Veto implements the FIP Decision with probability 1 when there is at least one private value agent $(\exists i$ s.t. 
$z_{i}=z_{P}$ ). In that case, the FIP Decision (i.e. $B$ ) is always chosen since $z_{P}$-agents cast a $v$ vote. The same is true under Unanimity but not under Majority. Second, when all agents have common values $\left(z_{i} \neq z_{P} \forall i\right)$, the FIP Decision coincides with the objective of common value agents: selecting $B$ in state $\omega_{B}$ and $R$ in state $\omega_{R}$. To understand why Veto performs better than Unanimity and Majority in that case, first notice that any strategy profile in Majority or Unanimity can be reproduced under Veto. Second, recall from McLennan (1998) that, in a pure common value environment, a strategy profile producing the maximal ex ante utility must be an equilibrium. Third, given Proposition 1, we have that, under Veto, common value agents behave as if they were playing the pure commonvalue game in which $\beta=0$. Finally, given that common value agents behave as if $\beta=0$, we have that the strategy profile that maximize the probability of selecting $B$ in state $\omega_{B}$ and $R$ in state $\omega_{R}$ when $z_{i} \neq z_{P} \forall i$ must be an equilibrium under Veto. Therefore, there must exist an equilibrium in Veto that implements the FIP Decision with a probability at least as high as the best equilibrium under either Majority or Unanimity.

Now, let us explain the strict dominance of Veto over Majority. When $\beta>0$, this is a direct implication of the reasoning above. For some realized type profiles (i.e. $\exists i$ s.t. $z_{i}=z_{P}$ ), the probability to implement the Pareto criterion is strictly lower under Majority than Veto. And for all possible realized type profiles, it cannot be greater. In expectations, it is therefore lower. When $\beta=0$, we are in a pure common value world. In that case, it is straightforward to show that the unique equilibrium under Majority corresponds to the equilibrium under Veto characterized in Proposition 2 if and only if $\phi_{B} \leq \phi_{3}$. In that case, the probability to implement the Pareto criterion is therefore identical under the two rules. When $\phi_{B}>\phi_{3}$, however, $v$ is always played with positive probability in equilibrium under Veto. By McLennan (1998), and with a reasoning similar to that above, the probability of taking the "wrong decision" must then be higher under Majority than Veto.

Before discussing the strict dominance of Veto over Unanimity, it is useful to note that when $\phi_{B} \geq \phi_{4}$ Unanimity aggregates information well. To see this, it is enough to consider the common value game. When $\phi_{B} \geq \phi_{4}$, a signal $s_{B}$ is relatively so precise that it suffices to convince a common value agent that the reform should be rejected, even if she is certain that all other agents have received an opposite signal. In such a case (which was 
not considered by Feddersen and Pesendorfer 1998), Unanimity performs of course better than Majority, and is in fact optimal (see below). Veto does equally well as Unanimity (agent behavior is isomorphic under the two rules), but can obviously not improve on this.

When $\phi_{B}<\phi_{4}$, Veto strictly dominates Unanimity because the latter does not aggregate information well (and both systems are equally good at protecting minorities). The poor performances of Unanimity in terms of information aggregation have been pointed out by Feddersen and Pesendorfer (1998). Essentially, the problem comes from the fact that the only way to "disclose" a signal $s_{B}$ is to veto the reform, which nails down the group decision. Agents are therefore reluctant to use it. Under Veto, it is possible to vote against the reform without nailing down the group decision, which solves the problem.

Theorem 1 has strong implications at the constitution writing stage. That is, from the point of view of a group that has to design its voting system before knowing the parameters $\phi_{B}, \phi_{R}, n$, and $\beta$. Imagine that there exist probability distribution functions over these parameters. Then, unless the probability to have $\beta=0$ and $\phi_{R} \leq \phi_{3}$ is equal to 1 (in which case we are in a pure common value game and there is no minority to protect), Veto strictly dominates Majority at the constitution writing stage. Also, unless $\phi_{B} \geq \phi_{4}$ with probability 1 (and Unanimity aggregates information well), Veto strictly dominates Unanimity at the constitution writing stage. If both probabilities are strictly below 1 , and there is therefore a relevant tension between information aggregation and minority protection, Veto should be strictly preferred to both.

\subsubsection{Optimality}

A voting system can be considered optimal if it implements with probability 1 the decision that would be taken by a benevolent dictator who observes the type profile (but not the state) and applies the Pareto criterion. Although Veto clearly outperforms Majority and Unanimity, it is not always optimal. In this subsection, we formally identify the range of parameter values for which Veto is optimal, and we describe a more elaborate system that is optimal for all values of the parameters. See the introduction for the reasons why we focus on Veto.

The following proposition identifies the range of parameter values for which Veto is an optimal voting system. 
Proposition 4 For the equilibrium characterized in Proposition 2, Veto is an optimal voting system if $\phi_{B} \in\left[\phi_{1}, \phi_{2}\right]$ or $\phi_{B} \geq \phi_{4}$.

Proof. Straightforward.

This result is obvious when there is at least one private value agent $\left(\exists i\right.$ s.t. $\left.z_{i}=z_{P}\right)$ : under Veto, the group rejects the reform, as would the benevolent dictator. We therefore focus on type profiles that do not include any private value agent $\left(z_{i} \neq z_{P} \forall i\right)$.

For $\phi_{B} \geq \phi_{4}$, a signal $s_{B}$ is relatively so precise, that it suffices to convince a common value agent that the reform should be rejected, even if it is certain that all other agents have received an opposite signal. Thus, under Veto, the group rejects the reform, for any type profile including a $z_{B}$-agent. This is the optimal decision. If the type profile does not include a $z_{B}$-agent, the reform is adopted under Veto. Again, this is the optimal decision.

To understand the optimality of Veto when $\phi_{B} \in\left[\phi_{1}, \phi_{2}\right]$, first notice that the benevolent dictator would choose $R$ if there are strictly more $z_{R}$-agents than $z_{B}$-agents, and $B$ otherwise (remember that we are focusing on type profile not including any private value agent). This is so because $s_{B}$ and $s_{R}$ signals have similar precision. Second, notice that, for the equilibrium described in Proposition 2, common value agents vote "sincerely" (i.e. $z_{B}$-agents vote $b$ and $z_{R}$-agents vote $r$ ) no matter the value of $\beta$ and $n$. This directly implies that the reform is adopted if and only if there are strictly more $z_{R}$-agents than $z_{B}$-agents. Veto is thus an optimal voting system. Note that Unanimity is also optimal in this region, but Majority performs very poorly.

The region where common value agents vote "sincerely" is intimately related to the majority threshold $q$ that is used under Veto. For simplicity, we have considered here a simple majority threshold, i.e. $q=n / 2$. However, note that for any given values of $\phi_{B}, \phi_{R}$, $n$, and $\beta$, there exists a majority threshold $q$ for which common value agents vote sincerely (see e.g. Austen-Smith and Banks 1996), and for which Veto is therefore optimal. Note that Majority is also optimal in this region, but here, it is Unanimity that performs very poorly.

When $n$ grows large, we can also prove the following optimality result:

Proposition 5 For the equilibrium characterized in Proposition 2, the probability of implementing the FIP Criterion converges to 1 when $n$ grows large. Veto is thus asymptoti- 
cally optimal for all $\phi_{B}, \phi_{R}$, and $\beta$.

Proof. See Appendix A2.

Do Unanimity and/or Majority share the same feature? The answer is no. There exist parameter values for which Unanimity implements the FIP Decision with a probability strictly lower than 1, even when n tends to infinity (see Feddersen and Pesendorfer 1998, for a proof that holds when $\phi_{R}=\phi_{B}$ and $\beta=0$ ). The problem is different for Majority. That system performs well when there are no $z_{P}$-agent. Yet, since $z_{P}$-agents do not have veto power, Majority implements the FIP Decision with probability strictly lower than 1 when $\exists i$ s.t. $z_{i}=z_{P}$.

Finally, we can prove that there exists the following two-round optimal voting system. In the first round, each voter casts a ballot $q_{i} \in\{0, \ldots, n\}$ (where $n$ is the size of the group) to suggest a supermajority threshold. If all $q_{i}$ are equal (say to $q$ ), then the rule used in the second round is a $q$-majority rule (i.e. the reform is implemented if and only if there are $q$ or more votes for $r$ ). Otherwise, the rule used in the second round is the $n$-majority rule (which is equivalent to Unanimity). That system generates the following efficient equilibrium (but it also generate many other non-efficient equilibria). In the second round, private value agents always vote $b$, and common value agent play the unique responsive symmetric equilibrium for the $q$-majority chosen in the first round. In the first round, private value agents choose the threshold $q=n$ and common value agents all choose the threshold $q$ that induces them to play sincerely in the second round conditional on $z_{i} \neq z_{P} \forall i$ (see e.g. Austen-Smith and Banks 1996).

\subsection{Interim Pareto Dominance}

In this section, we use the concept of Interim Pareto dominance proposed by Holmstrom and Myerson (1983) to derive even stronger results on the comparison between Veto and Unanimity. In short, a system Interim Pareto Dominates another if it makes all agents better off from an interim standpoint.

The concept of Interim Pareto Dominance, relies on the notion of interim utility that

we have not formally defined yet. Remember that the (von Neumann-Morgenstern) utility of an agent of type $z$ for decision $D$ in state $\omega$ is $u_{z}(D, \omega)$. This utility, when evaluated 
under voting system $\Psi$, for the equilibrium strategy profile $\sigma^{* \Psi}$, for the realized type profile $\mathbf{z}$, in state $\omega$, corresponds to $u_{z}\left(D\left(\sigma^{* \Psi}, \mathbf{z}\right), \omega\right)$. Taking the expectation of the utility (conditional on the agent's type $z$ ) with respect to $\omega$ and $\mathbf{z}$, we obtain the interim utility of an agent of type $z$ under voting system $\Psi$ :

$$
E_{\mathbf{z}, \omega}\left[u_{z}\left(D\left(\sigma^{* \Psi}, \mathbf{z}\right), \omega\right)\right]
$$

And we can now formally define the concept of Interim Pareto dominance:

Definition 5 A voting system $\Psi$ Interim Pareto dominates (IP dominates) a voting system $\Psi^{\prime}$ if there exists an equilibrium $\sigma^{* \Psi}$ under $\Psi$ such that: $E_{\mathbf{z}, \omega}\left[u_{z}\left(D\left(\sigma^{* \Psi}, \mathbf{z}\right), \omega\right)\right] \geq$ $E_{\mathbf{z}, \omega}\left[u_{z}\left(D\left(\sigma^{* \Psi^{\prime}}, \mathbf{z}\right), \omega\right)\right]$, for all $\sigma^{* \Psi^{\prime}}, z$, and $\Psi$ strictly IP dominates $\Psi^{\prime}$ if this condition also holds with strict inequality for some $z$.

We have established that when $\phi_{B} \geq \phi_{4}$, the outcomes under Veto and Unanimity are identical, and that, when $\phi_{B}<\phi_{4}$, Veto strictly FIP dominates Unanimity. We can establish a stronger result when $\phi_{B}<\phi_{4}$.

Theorem 2 For all $\beta$ and $n$, Veto IP dominates Unanimity, and, unless $\phi_{B} \geq \phi_{4}$, Veto strictly IP dominates Unanimity.

Proof. See Appendix A2.

Let us focus on $\phi_{B}<\phi_{4}$ and separately consider the case of private and common value agents. First, private value agents are indifferent since they can and do enforce $B$ under both systems. When there is a private value agent (i.e. $\exists i$ s.t. $z_{i}=z_{P}$ ), common value agents are therefore equally well off under both systems. When there are none (i.e. $z_{i} \neq z_{P}$ $\forall i$ ), let us consider agents $z_{B}$ and $z_{R}$ in turn. Since agents $z_{B}$ always play $v$ with positive probability under $U$, their interim utility equals the utility of getting $B$ with probability 1. Since they can also play $v$ under Veto (and get $B$ with probability 1 ), they cannot be worse off. By a simple revealed preference argument, they are strictly better off when they strictly prefer to vote $b$. For $z_{R}$-agents the intuition goes as follows: under $U$ the reform $R$ is rarely chosen (Unanimity is "the" gridlock system). This implies that the probability of making a mistake is "very" likely in state $\omega_{R}$. Given that $z_{R}$-agents believe that state 
$\omega_{R}$ is more likely than state $\omega_{B}$, their interim utility under $U$ is low. Since $V$ does not suffer from the same weakness, $z_{R}$-agents are strictly better off under $V$ than under $U$.

Theorem 2 is a powerful result. First because it implies that (i) no agent (even after learning their private information) would object to get rid of Unanimity and use Veto instead, and (ii) except in cases of lesser relevance, some would strictly prefer to use Veto. In the wording of Holmstrom and Myerson (1983), Unanimity is not a durable voting system. Second, Theorem 2 has implications for the welfare comparison between Veto and Unanimity that are even stronger than FIP dominance. To see this, consider a weighted utilitarian social welfare function that assigns arbitrary positive weights to the interim utility of the three possible types of agents (i.e. $z_{P}, z_{B}$, and $z_{R}$ ).

Corollary 1 For all $\phi_{B}, \phi_{R}, \beta, n$, weighted utilitarian social welfare is at least has high under Veto than under Unanimity. Unless $\phi_{B} \geq \phi_{4}$, it is strictly higher under Veto than under Unanimity

The intuition for this striking result is rather simple: since granting veto power to agents allows them to protect their own interests as well as under Unanimity, the relevant dimension for comparing Veto and Unanimity is information aggregation. And Veto does at least as well as Majority on that dimension.

Corollary 1 makes very clear why Interim Pareto Dominance is a stronger result than Full Information Pareto Dominance. By construction, the latter cannot account for the relative social cost of not implementing the Pareto criterion in different circumstances. All that matters is the ex ante probability to implement the Pareto criterion. By contrast, the weights in the social welfare function can be interpreted as the relative social importance of the minority protection motive and the information aggregation motive. And what Corollary 1 says is that, no matter the weights, welfare is higher under Veto than Unanimity.

\section{Conclusion}

Finding a voting system that implements the Pareto criterion is challenging because of the potential tension between minority protection and information aggregation. In this paper, we analyzed a voting system that essentially resolves this tension: majority rule 
with veto power (Veto). We compared Veto to two commonly used voting systems: (i) Unanimity, which is known for protecting even the smallest minority, and (ii) Majority, which is known for aggregating information well.

We proved three main results. First, we characterized the set of equilibria under Veto and argued that only one equilibrium is relevant. Second, we proved that Veto implements with higher ex ante probability than Majority and Unanimity the full information Pareto criterion. Third, we used the concept of Interim Pareto Dominance (Holmstron and Myerson 1983) to derive even stronger results on the comparison between Veto and Unanimity: even once types are realized, no agent can be worse off under Veto than Unanimity, and (except in some cases of lesser relevance) some agents are strictly better off.

Our results shed a new light on the evolution of voting procedures in EU institutions since the Treaty of Maastricht. In 1992, that Treaty established Unanimity as the default procedure of the European Council. ${ }^{17}$ In 1997, the Treaty of Amsterdam introduced the concept of "constructive abstention", which allows abstentions in the European Council (when presiding over the Common Foreign and Security Policy) without blocking a unanimous decision unless more than $1 / 3$ of the Member States abstain. ${ }^{18}$ Our results suggest that this change may reflect the dual desire of the Union to protect the private interests of the Member States without impairing information aggregation. ${ }^{19}$ Indeed, unanimity rule augmented with constructive abstention is closely related to Veto. To see this, notice that Veto is equivalent to a modified version of Unanimity where abstention is allowed, and a majority approval quorum is required for the reform to be adopted. Re-labelling the $b$-ballot under Veto as "abstention" makes that equivalence obvious.

In our view, in addition of its strong theoretical properties, Veto's simplicity makes it particularly appealing to real-world applications. And indeed, there are voting bodies that use this voting system or slight variations thereof (e.g. the UN Security Council and

\footnotetext{
${ }^{17}$ According to Wessel and Bottner (2013), the main reason underlying that choice is the participation of Member States. For instance, they claim that: "It is safe to assume that the inclusion of the CFSP in the 1992 Maastricht Treaty was possible only because of the absence of majority voting." (Wessel and Bottner, 2013, p. 5).

${ }^{18}$ Note that this approval quorum is binding only when Member States accompany the abstention by a formal declaration. In that case, the Member States who abstain are not obliged to apply the decision but must accept that it commits the Union, and must then refrain from any action that might conflict with Union action based on that decision. Wessel and Bottner (2013) argue that the latter provision makes essentially void the option not to apply the decision.

${ }^{19}$ Wessel and Bottner (2013) argue that the introduction of constructive abstention was indeed motivated by the understanding that Unanimity was impeding progress on the Common Foreign and Security Policy.
} 
the European Council). Still, there are also cases where voting bodies use the unanimity rule without allowing for abstention and/or imposing an approval quorum. Three notable examples are those of American juries, voting bodies of the Southern Common Market (Mercosur), and the North Atlantic Treaty Organization (NATO). ${ }^{20}$ Feddersen and Pesendorfer (1998) have argued that such a system has very bad information aggregation properties and may lead, contrarily to common wisdom, to a relatively high rate of type I errors (e.g. conviction of innocent defendants). Our theoretical results suggest that allowing for abstention and an approval quorum would solve this problem. Furthermore, since Veto is a relatively simple system and it Interim Pareto dominates Unanimity, such an institutional reform should in theory not be too difficult to implement. In practice, one might however argue that Veto is more complex than Unanimity (since the set of actions is larger). Such additional complexity, if badly handled by real world individuals, could weaken Veto or even overturn its superiority. We address these questions in a companion paper, Bouton et al. (2014), through a series of controlled laboratory experiments. Overall, we find strong support for the dominance of Veto over both Majority and Unanimity.

\footnotetext{
${ }^{20}$ Note that Mercosur and NATO decisions are made by Consensus, i.e. a reform is adopted if and only if no member vetoes it. As discussed in Section 2, Consensus and Unanimity are strategically equivalent.
} 


\section{References}

[1] Ali, N., Goeree, J., Kartik, N. and T. Palfrey (2008). Information Aggregation in Ad Hoc and Standing Committees, American Economic Review, Papers \& Proceedings, 98(2): 181-186.

[2] Austen-Smith, D. and J. Banks (1996). Information Aggregation, Rationality, and the Condorcet Jury Theorem, American Political Science Review, 90: 34-45.

[3] Austen-Smith, D. and T. Feddersen (2006). Deliberation, Preference Uncertainty and Voting Rules, American Political Science Review, 100(2): 209-217.

[4] Battaglini, M., R. Morton, and T. Palfrey (2008). Information Aggregation and Strategic Abstention in Large Laboratory Elections, American Economic Review, Papers \& Proceedings, 98(2): 194-200.

[5] Battaglini, M., Morton, R. and T. Palfrey (2010). The Swing Voter's Curse in the Laboratory, Review of Economic Studies, 77: 61-89.

[6] Bhattacharya, S., J. Duffy and S. T. Kim (2012). Compulsory versus Voluntary Voting: An Experimental Study, mimeo.

[7] Blum, Y.Z. (2005). Proposals for UN Security Council Reform, American Journal of International Law, 99(3): 632-649.

[8] Bond, P. and H. Eraslan (2009). Strategic Voting over Strategic Proposals, Review of Economic Studies, 77(2): 459-490.

[9] Bouton, L. (2013). A Theory of Strategic Voting in Runoff Elections, American Economic Review, 103(4): 1248-1288.

[10] Bouton, L., and M. Castanheira (2012). One Person, Many Votes: Divided Majority and Information Aggregation, Econometrica, 80(1): 43-87.

[11] Bouton, L., M. Castanheira, and A. Llorente-Saguer (2012). Divided Majority and Information Aggregation: Theory and Experiment, IED Discussion Paper, 242. 
[12] Bouton, L., A. Llorente-Saguer, and F. Malherbe (2014). Vetos, Abstention and Frames: An Experimental Study on the Implementation of Majority with Veto Power, mimeo.

[13] Buchanan, J.M. and Tullock, G. (1962). The Calculus of Consent. Ann Arbor: University of Michigan Press.

[14] Cameron, C.M. (2000). Veto Bargaining: Presidents and the Politics of Negative Power, Cambridge University Press.

[15] Chen, Y. and P. Ordeshook (1998). Veto Games: Spatial Committees under Unanimity Rule, Public Choice, 97(4): 617-643.

[16] Chwe, M. (1999). Minority Voting Rights Can Maximize Majority Welfare, American Political Science Review, 93(1): 85-97.

[17] Coughlan, P. (2001). In Defense of Unanimous Jury Verdicts: Mistrials, Communication and Strategic Voting, American Political Science Review, 94: 375-93.

[18] Dougherty, K. and J. Edward (2005). A Nonequilibrium Analysis of Unanimity Rule, Majority Rule, and Pareto. Economic Inquiry, 43(4): 855-864.

[19] Feddersen, T. and W. Pesendorfer (1996). The Swing Voter's Curse, American Economic Review, 86: 408-424.

[20] Feddersen, T. and W. Pesendorfer (1997). Voting Behavior and Information Aggregation in Elections with Private Information, Econometrica, 65: 1029-1058.

[21] Feddersen, T. and W. Pesendorfer (1998). Convicting the Innocent: the Inferiority of Unanimous Jury Verdicts, American Political Science Review, 92: 23-35.

[22] Gerardi, D. and L. Yariv (2007). Deliberative Voting, Journal of Economic Theory, 114: $357-394$.

[23] Gerardi, D. and L. Yariv (2008). Information Acquisition in Committees, Games and Economic Behavior, 62: 436-459.

[24] Goeree, J. and L. Yariv (2011). An Experimental Study of Collective Deliberation, Econometrica, 79(3): 893-921. 
[25] Groseclose, T. and N. McCarty (2001). The Politics of Blame: Bargaining Before an Audience, American Journal of Political Science, 45(1): 100-119.

[26] Guarnaschelli, S., R. McKelvey and T. Palfrey (2000). An Experimental Study of Jury Decision Rules, American Political Science Review, 94(2): 407-423.

[27] Guttman, J. (1998). Unanimity and Majority Rule: the Calculus of Consent Reconsidered, European Journal of Political Economy, 14: 189-207.

[28] Holmstrom, B. and R. Myerson (1983). Efficient and Durable Decision Rules with Incomplete Information, Econometrica, 51(6): 1799-1819.

[29] Maggi, G. and M. Morelli (2006). Self-Enforcing Voting in International Organizations, American Economic Review, 96(4): 1137-1158.

[30] Martinelli, C. (2006). Would Rational Voters Acquire Costly Information?, Journal of Economic Theory, 129: 225-251.

[31] Martinelli, C. (2007). Rational Ignorance and Voting Behavior, International Journal of Game Theory, 35: 315-335.

[32] McLennan, A. (1998). Consequences of the Condorcet Jury Theorem for Beneficial Information Aggregation by Rational Agents, American Political Science Review, 92: 413-418.

[33] McMurray, J. (2013). Aggregating Information by Voting: The Wisdom of the Experts versus the Wisdom of the Masses, Review of Economic Studies, 80(1): 277-312.

[34] Myerson, R. (1998). Population Uncertainty and Poisson Games, International Journal of Game Theory, 27: 375-392.

[35] Myerson, R. (2000). Large Poisson Games, Journal of Economic Theory, 94: 7-45.

[36] Myerson, R. (2002). Comparison of Scoring Rules in Poisson Voting Games, Journal of Economic Theory, 103: 219-251.

[37] Myerson, R. and R. Weber (1993). A Theory of Voting Equilibria, American Political Science Review, 87: 102-114. 
[38] Nunnari, S. (2012). Dynamic Legislative Bargaining with Veto Power, mimeo.

[39] Persico, N. (2004). Committee Design with Endogenous Information, Review of Economic Studies, 71: 165-191.

[40] Romme, A. G. L. (2004). Unanimity Rule and Organizational Decision Making: A Simulation Model, Organization Science, 15(6): 704-718.

[41] Sobel, R. and R. Holcombe (2001). The Unanimous Voting Rule Is Not the Political Equivalent to Market Exchange, Public Choice, 106: 233-242.

[42] Tsebelis, G. (2002). Veto Players: How Political Institutions Work, Princeton New Jersey: Princeton University Press.

[43] Van Weelden, R. (2008). Deliberation Rules and Voting, Quarterly Journal of Political Science, 3: 83-88.

[44] Wessel, R. and R. Bottner (2013). The Procedures Leading to the Adoption of Decisions in the CFSP Area - Art. 31 TEU. In H.-J. Blanke and S. Mangiameli (eds.), The Treaty on European Union (TEU): A Commentary, Berlin/Heidelberg: Springer.

[45] Wicksell, K. (1967 [1896]). A New Principle of Just Taxation. In R.A. Musgrave and A.T. Peacocks (eds.), Classics in the Theory of Public Finance, 72-118. New York: St. Martin's Press.

[46] Winter, E. (1996). Voting and Vetoing, American Political Science Review, 4: 813823.

[47] Woods, N. (2000). The Challenge of Good Governance for the IMF and the World Bank Themselves, World Development, 28(5): 823-841. 


\section{Appendices}

\section{Appendix A1: Proofs of Section 3}

Proof of Proposition 1. First, recall that $v$ is a strictly dominant strategy for $z_{P}$-agent. Therefore, any responsive equilibrium under Veto satisfies point (i) in the Proposition.

Second, given point (i), if $\exists i$ s.t. $z_{i}=z_{P}$, the payoffs to the other agents are not affected by their behavior, for the group decision is $B$ no matter what they do. Hence, all common value agents condition their behavior on $z_{i} \neq z_{P} \forall i$. Therefore, we have that (1) the strategy profile played by common-value agents in any equilibrium under Veto must form an equilibrium when $\beta=0$.(2) if $\sigma^{*}$ is an equilibrium strategy profile of the game when $\beta=0$, it must be an optimal strategy profile for common value agents $\forall \beta$.

Proof of Proposition 2. Let us start by collecting the equations defining the gains to vote $r$ or $v$ for each common value agent type:

$$
\begin{aligned}
& G\left(r \mid z_{R}\right)=\frac{\phi_{R}}{\phi_{R}+1}\left(\begin{array}{c}
n-1 \\
n-1
\end{array}\right)\left(\tau_{r}^{\omega_{R}}\right)^{\frac{n-1}{2}}\left(\tau_{b}^{\omega_{R}}\right)^{\frac{n-1}{2}}-\frac{1}{\phi_{R}+1}\left(\begin{array}{c}
n-1 \\
n-1
\end{array}\right)\left(\tau_{r}^{\omega_{B}}\right)^{\frac{n-1}{2}}\left(\tau_{b}^{\omega_{B}}\right)^{\frac{n-1}{2}}, \\
& G\left(r \mid z_{B}\right)=\frac{1}{\phi_{B}+1}\left(\begin{array}{c}
n-1 \\
\frac{n-1}{2}
\end{array}\right)\left(\tau_{r}^{\omega_{R}}\right)^{\frac{n-1}{2}}\left(\tau_{b}^{\omega_{R}}\right)^{\frac{n-1}{2}}-\frac{\phi_{B}}{\phi_{B}+1}\left(\begin{array}{c}
n-1 \\
\frac{n-1}{2}
\end{array}\right)\left(\tau_{r}^{\omega_{B}}\right)^{\frac{n-1}{2}}\left(\tau_{b}^{\omega_{B}}\right)^{\frac{n-1}{2}}, \\
& G\left(v \mid z_{R}\right)=\frac{1}{\phi_{R}+1} \sum_{j=\frac{n+1}{2}}^{n-1}\left(\begin{array}{c}
n-1 \\
j
\end{array}\right)\left(\tau_{r}^{\omega_{B}}\right)^{j}\left(\tau_{b}^{\omega_{B}}\right)^{n-1-j}-\frac{\phi_{R}}{\phi_{R}+1} \sum_{j=\frac{n+1}{2}}^{n-1}\left(\begin{array}{c}
n-1 \\
j
\end{array}\right)\left(\tau_{r}^{\omega_{R}}\right)^{j}\left(\tau_{b}^{\omega_{R}}\right)^{n-1-j}, \\
& G\left(v \mid z_{B}\right)=\frac{\phi_{B}}{\phi_{B}+1} \sum_{j=\frac{n+1}{2}}^{n-1}\left(\begin{array}{c}
n-1 \\
j
\end{array}\right)\left(\tau_{r}^{\omega_{B}}\right)^{j}\left(\tau_{b}^{\omega_{B}}\right)^{n-1-j}-\frac{1}{\phi_{B}+1} \sum_{j=\frac{n+1}{2}}^{n-1}\left(\begin{array}{c}
n-1 \\
j
\end{array}\right)\left(\tau_{r}^{\omega_{R}}\right)^{j}\left(\tau_{b}^{\omega_{R}}\right)^{n-1-j},
\end{aligned}
$$

where:

$$
\begin{aligned}
& \tau_{r}^{\omega}=\left(1-\epsilon^{*}-\gamma^{*}\right) \operatorname{Pr}\left(z_{R} \mid \omega\right)+\eta^{*} \operatorname{Pr}\left(z_{B} \mid \omega\right) \\
& \tau_{b}^{\omega}=\left(\epsilon^{*}+\gamma^{*}\right) \operatorname{Pr}\left(z_{R} \mid \omega\right)+\left(1-\eta^{*}-\delta^{*}\right) \operatorname{Pr}\left(z_{B} \mid \omega\right) \\
& \tau_{v}^{\omega}=\delta^{*} \operatorname{Pr}\left(z_{B} \mid \omega\right) .
\end{aligned}
$$

The proof consists of five parts, each corresponding to an element of the partition in the proposition.

Part (i): The first threshold is $\phi_{1} \equiv\left(\phi_{R}\right)^{\frac{n-1}{n+1}}$. When. $\phi_{B} \in\left(1, \phi_{1}\right)$, we have $\eta^{*} \in(0,1)$ and $\epsilon^{*}=\delta^{*}=\gamma^{*}=0$.

Collecting the actions played with strictly positive probability, we have: $\sigma_{r}\left(z_{R}\right)=1, \sigma_{r}\left(z_{B}\right)=$ $\eta^{*}$, and $\sigma_{b}\left(z_{B}\right)=1-\eta^{*}$. For this to be an equilibrium, we need: (i.a) $G\left(r \mid z_{R}\right) \geq 0$, (i.b) $G\left(r \mid z_{B}\right)=$ 0, (i.c) $G\left(v \mid z_{B}\right) \leq 0$, and (i.d) $G\left(v \mid z_{R}\right) \leq 0$. From $G\left(r \mid z_{R}\right) \geq G\left(r \mid z_{B}\right)$ and condition (i.b), we have that (i.a) is necessarily satisfied. From $G\left(v \mid z_{B}\right) \geq G\left(v \mid z_{R}\right)$ and (i.c), we have that (i.d) is necessarily satisfied. It remains to prove that conditions (i.b) and (i.c) are satisfied.

We start by discussing condition (i.b). In this case, we have that $G\left(r \mid z_{B}\right)=0$ if

$$
\phi_{B}^{\left(\frac{n+1}{n-1}\right)}=\left(\frac{\phi_{R}\left(\phi_{B}-1\right)+\eta^{*}\left(\phi_{R}-1\right)}{\left(\phi_{B}-1\right)+\eta^{*} \phi_{B}\left(\phi_{R}-1\right)}\right) .
$$


Solving for $\eta^{*}$ gives

$$
\eta^{*}=\frac{\left(\phi_{B}-1\right)\left(\phi_{R}-\left(\phi_{B}\right)^{\frac{n+1}{n-1}}\right)}{\left(\phi_{R}-1\right)\left(\left(\phi_{B}\right)^{\frac{2 n}{n-1}}-1\right)} .
$$

Since $\phi_{B}, \phi_{R}>1$, the denominator is positive. Given that we consider $\phi_{B}<\phi_{1}$, the numerator is positive as well. Since $\phi_{B} \phi_{R}>1$, the numerator is strictly smaller than the denominator. Therefore, there exists a unique $\eta^{*} \in(0,1)$ such that this condition is satisfied.

We now discuss condition (i.c). In this case, $G\left(v \mid z_{B}\right) \leq 0$ reads

$$
\phi_{B}\left(\sum_{j=\frac{n+1}{2}}^{n-1}\left(\begin{array}{c}
n-1 \\
j
\end{array}\right)\left(\tau_{r}^{\omega_{B}}\right)^{j}\left(\tau_{b}^{\omega_{B}}\right)^{n-1-j}\right) \leq \sum_{j=\frac{n+1}{2}}^{n-1}\left(\begin{array}{c}
n-1 \\
j
\end{array}\right)\left(\tau_{r}^{\omega_{R}}\right)^{j}\left(\tau_{b}^{\omega_{R}}\right)^{n-1-j}
$$

A sufficient condition for this to hold is that it holds term-by-term. In particular, the condition holds if $\forall j \in\left\{\frac{n+1}{2}, \ldots, n-1\right\}$, we have

$$
\phi_{B}\left(\begin{array}{c}
n-1 \\
j
\end{array}\right)\left(\tau_{r}^{\omega_{B}}\right)^{j}\left(\tau_{b}^{\omega_{B}}\right)^{n-1-j} \leq\left(\begin{array}{c}
n-1 \\
j
\end{array}\right)\left(\tau_{r}^{\omega_{R}}\right)^{j}\left(\tau_{b}^{\omega_{R}}\right)^{n-1-j} .
$$

In the present case, this boils down to

$$
\phi_{B} \leq\left(\frac{\phi_{R}\left(\phi_{B}-1\right)+\eta^{*}\left(\phi_{R}-1\right)}{\left(\phi_{B}-1\right)+\eta^{*} \phi_{B}\left(\phi_{R}-1\right)}\right)^{\frac{j}{n-j}} .
$$

But, we know from $G\left(r \mid z_{B}\right)=0$ that

$$
\phi_{B}=\left(\frac{\phi_{R}\left(\phi_{B}-1\right)+\eta^{*}\left(\phi_{R}-1\right)}{\left(\phi_{B}-1\right)+\eta^{*} \phi_{B}\left(\phi_{R}-1\right)}\right)^{\frac{n-1}{n+1}}
$$

Given $\frac{\phi_{R}\left(\phi_{B}-1\right)+\eta^{*}\left(\phi_{R}-1\right)}{\left(\phi_{B}-1\right)+\eta^{*} \phi_{B}\left(\phi_{R}-1\right)} \geq 1$, and $\frac{j}{n-j} \geq \frac{n+1}{n-1}>\frac{n-1}{n+1}, \forall j \in\left\{\frac{n+1}{2}, \ldots, n-1\right\}$, we have that the condition must be satisfied.

Part (ii): The second threshold is $\phi_{2} \equiv\left(\phi_{R}\right)^{\frac{n+1}{n-1}}$. When $\phi_{B} \in\left[\phi_{1}, \phi_{2}\right]$, we have: $\eta^{*}=\epsilon^{*}=\delta^{*}=$ $\gamma^{*}=0$.

For $\sigma_{r}\left(z_{R}\right)=1$, and $\sigma_{b}\left(z_{B}\right)=1$ to be an equilibrium, we need (ii.a) $G\left(r \mid z_{R}\right) \geq 0$, (ii.b) $G\left(r \mid z_{B}\right) \leq$ 0, (ii.c) $G\left(v \mid z_{B}\right) \leq 0$, and (ii.d) $G\left(r \mid z_{R}\right) \geq G\left(v \mid z_{R}\right)$. From $G\left(v \mid z_{B}\right) \geq G\left(v \mid z_{R}\right)$ and (ii.a) and (ii.c), we have that (ii.d) is necessarily satisfied. It remains to prove that conditions (ii.a), (ii.b), and (ii.c) are satisfied.

First, we discuss condition (ii.a). In this case, $G\left(r \mid z_{R}\right) \geq 0$ boils down to

$$
\phi_{R} \geq\left(\frac{1}{\phi_{R}}\right)^{\frac{n-1}{2}}\left(\phi_{B}\right)^{\frac{n-1}{2}},
$$

which is satisfied iff $\phi_{B} \leq \phi_{2}$, and is therefore satisfied here. 
Second, we discuss condition (ii.b). In this case, $G\left(r \mid z_{B}\right) \leq 0$ boils down to

$$
\frac{1}{\phi_{B}} \leq\left(\frac{1}{\phi_{R}}\right)^{\frac{n-1}{2}}\left(\phi_{B}\right)^{\frac{n-1}{2}}
$$

which is satisfied iff $\phi_{B} \geq \phi_{1}=\left(\phi_{R}\right)^{\frac{n-1}{n+1}}$, and is therefore satisfied here.

Finally, we discuss condition (ii.c). Following an identical approach as in Part (i) for the same condition, we obtain a term-by-term sufficient condition for $G\left(v \mid z_{B}\right) \leq 0$ :

$$
\phi_{B} \leq\left(\phi_{R}\right)^{\frac{j}{n-j}} .
$$

From $\phi_{B} \leq \phi_{2}=\left(\phi_{R}\right)^{\frac{n+1}{n-1}}, \phi_{R}>1$, and $\frac{j}{n-j} \geq \frac{n+1}{n-1} \forall j \in\left\{\frac{n+1}{2}, \ldots, n-1\right\}$, we have that this is satisfied.

Part (iii): When $\phi_{B} \in\left(\phi_{2}, \phi_{3}\right]$, we have: $\epsilon^{*} \in(0,1)$ and $\eta^{*}=\delta^{*}=\gamma^{*}=0$.

For $\sigma_{r}\left(z_{R}\right)=1-\epsilon^{*}, \sigma_{b}\left(z_{R}\right)=\epsilon^{*}$, and $\sigma_{b}\left(z_{B}\right)=1$ to be an equilibrium, we need (iii.a) $G\left(r \mid z_{R}\right)=$ 0, (iii.b) $G\left(r \mid z_{B}\right) \leq 0$, (iii.c) $G\left(v \mid z_{B}\right) \leq 0$, and (iii.d) $G\left(r \mid z_{R}\right) \geq G\left(v \mid z_{R}\right)$. From $G\left(v \mid z_{B}\right) \geq G\left(v \mid z_{R}\right)$, (iii.a), and (iii.c), we have that (iii.d) is necessarily satisfied. From $G\left(r \mid z_{B}\right) \leq G\left(r \mid z_{R}\right)$, and (iii.a), we have that (iii.b) is necessarily satisfied. It remains to prove that conditions (iii.a) and (iii.c) are satisfied.

First, we discuss condition (iii.a). $G\left(r \mid z_{R}\right)=0$ boils down to

$$
\phi_{R}=\left(\frac{1}{\phi_{R}}\right)^{\frac{n-1}{2}}\left(\frac{\epsilon^{*}\left(\phi_{B}-1\right)+\phi_{B}\left(\phi_{R}-1\right)}{\epsilon^{*} \phi_{R}\left(\phi_{B}-1\right)+\left(\phi_{R}-1\right)}\right)^{\frac{n-1}{2}} \text {, }
$$

and thus to

$$
\epsilon^{*}=\frac{\left(\phi_{R}-1\right)\left(\phi_{B}-\left(\phi_{R}\right)^{\frac{n+1}{n-1}}\right)}{\left.\left(\phi_{B}-1\right)\left(\left(\phi_{R}\right)^{\frac{n+1}{n-1}} \phi_{R}-1\right)\right)} .
$$

Since $\phi_{B}, \phi_{R}>1$, the denominator is strictly positive. Given that we consider $\phi_{B}>\phi_{2}$, the numerator is positive as well. Since $\phi_{B} \phi_{R}>1$, the numerator is strictly smaller than the denominator. Therefore, there exists a unique $\epsilon^{*} \in(0,1)$ such that this condition is satisfied.

Second, we implicitly define the third threshold $\phi_{3}$ as the unique value that satisfies:

$$
\phi_{3}=\phi_{R}^{n-1} \frac{\sum_{j=\frac{n+1}{2}}^{n-1}\left(\begin{array}{c}
n-1 \\
j
\end{array}\right)\left(1-\epsilon^{*}\right)^{j}\left(\phi_{3}-1\right)^{j}\left(\epsilon^{*}\left(\phi_{3}-1\right)+\frac{\left(\phi_{R}-1\right)}{\phi_{R}}\right)^{n-1-j}}{\sum_{j=\frac{n+1}{2}}^{n-1}\left(\begin{array}{c}
n-1 \\
j
\end{array}\right)\left(1-\epsilon^{*}\right)^{j}\left(\phi_{3}-1\right)^{j}\left(\epsilon^{*}\left(\phi_{3}-1\right)+\phi_{3}\left(\phi_{R}-1\right)\right)^{n-1-j}} .
$$

where $\epsilon^{*}=\bar{\epsilon}$, if $\bar{\epsilon}=\frac{\phi_{3}\left(1-\phi_{R}\right)-\left(\phi_{R}\right)^{\frac{n+1}{n-1}}\left(1-\phi_{R}\right)}{\phi_{R}\left(1-\phi_{3}\right)-\phi_{R}^{-\frac{n+1}{n-1}}\left(1-\phi_{3}\right)} \in[0,1]$ and $\epsilon^{*}=0$ otherwise. (See Part (iv) below for the proof that $\phi_{3}$ is unique.)

The condition above corresponds, by construction, to $G\left(v \mid z_{B}\right)=0$, when $\sigma_{b}\left(z_{R}\right)=\epsilon^{*}$, and $\sigma_{b}\left(z_{B}\right)=1$. Condition (iii.c) corresponds then to $\phi_{B} \leq \phi_{3}$ and is therefore satisfied . 
Part (iv): The fourth threshold is $\phi_{4} \equiv\left(\phi_{R}\right)^{n-1}$. When $\phi_{B} \in\left(\phi_{3}, \phi_{4}\right)$, we have that: $\delta^{*} \in$ $(0,1), \gamma^{*} \in\left(0,1-\delta^{*}\right)$ and $\eta^{*}=\epsilon^{*}=0$.

For $\sigma_{r}\left(z_{R}\right)=1-\gamma^{*}, \sigma_{b}\left(z_{R}\right)=\gamma^{*}, \sigma_{b}\left(z_{B}\right)=1-\delta^{*}$, and $\sigma_{v}\left(z_{B}\right)=\delta^{*}$ to be an equilibrium, we need (iv.a) $G\left(r \mid z_{R}\right)=0$, (iv.b) $G\left(v \mid z_{B}\right)=0$, (iv.c) $G\left(r \mid z_{R}\right) \geq G\left(v \mid z_{R}\right)$, and (iv.d) $G\left(v \mid z_{B}\right) \geq$ $G\left(r \mid z_{B}\right)$. From $G\left(v \mid z_{B}\right) \geq G\left(v \mid z_{R}\right)$, (iv.a), and (iv.b), condition (iv.c) is necessarily satisfied. From $G\left(r \mid z_{R}\right) \geq G\left(r \mid z_{B}\right)$, (iv.a), and (iv.b), condition (iv.d) is necessarily satisfied. It remains to prove that conditions (iv.a) and (iv.b) are satisfied.

First, we discuss condition (iv.a). In this case, $G\left(r \mid z_{R}\right)=0$ simplifies to

$$
\left(\frac{\left(1-\gamma^{*}\right)}{\left(1-\gamma^{*}\right) \phi_{R}}\right)^{\frac{n-1}{2}}\left(\frac{\gamma^{*}\left(\phi_{B}-1\right)+\left(1-\delta^{*}\right) \phi_{B}\left(\phi_{R}-1\right)}{\gamma^{*} \phi_{R}\left(\phi_{B}-1\right)+\left(1-\delta^{*}\right)\left(\phi_{R}-1\right)}\right)^{\frac{n-1}{2}}=\phi_{R},
$$

and thus to

$$
\frac{\gamma^{*}}{1-\delta^{*}}=\frac{\left(\phi_{R}-1\right)\left(\phi_{B}-\left(\phi_{R}\right)^{\frac{n+1}{n-1}}\right)}{\left(\phi_{B}-1\right)\left(\left(\phi_{R}\right)^{\frac{n+1}{n-1}} \phi_{R}-1\right)} \text {. }
$$

Since the RHS is equal to $\epsilon^{*}$, we have from part (iii) that $\frac{\gamma^{*}}{1-\delta^{*}} \in(0,1)$ iff $\phi_{B}>\left(\phi_{R}\right)^{\frac{n+1}{n-1}}=\phi_{2}$, which is satisfied in this case.

Second, we discuss condition (iv.b). In this case, $G\left(v \mid z_{B}\right)=0$ reads

$$
\phi_{B}=\frac{\sum_{j=\frac{n+1}{2}}^{n-1} \frac{(n-1) !}{j !(n-1-j) !}\left(\left(1-\gamma^{*}\right) \phi_{R}\left(\phi_{B}-1\right)\right)^{j}\left(\gamma^{*} \phi_{R}\left(\phi_{B}-1\right)+\left(1-\delta^{*}\right)\left(\phi_{R}-1\right)\right)^{n-1-j}}{\sum_{j=\frac{n+1}{2}}^{n-1} \frac{(n-1) !}{j !(n-1-j) !}\left(\left(1-\gamma^{*}\right)\left(\phi_{B}-1\right)\right)^{j}\left(\gamma^{*}\left(\phi_{B}-1\right)+\left(1-\delta^{*}\right) \phi_{B}\left(\phi_{R}-1\right)\right)^{n-1-j}} .
$$

or

$$
\frac{\phi_{B} \sum_{j=\frac{n+1}{2}}^{n-1}\left(\begin{array}{c}
n-1 \\
j
\end{array}\right)\left(1-\gamma^{*}\right)^{j}\left(\gamma^{*}+\left(1-\delta^{*}\right) \phi_{B} \frac{\phi_{R}-1}{\phi_{B}-1}\right)^{n-1-j}}{\phi_{R}^{n-1} \sum_{j=\frac{n+1}{2}}^{n-1}\left(\begin{array}{c}
n-1 \\
j
\end{array}\right)\left(1-\gamma^{*}\right)^{j}\left(\gamma^{*}+\left(1-\delta^{*}\right) \frac{\phi_{R}-1}{\phi_{R}\left(\phi_{B}-1\right)}\right)^{n-1-j}}=1
$$

We need to show that for each $\phi_{B} \in\left(\phi_{3}, \phi_{4}\right)$, there exist unique values for $\delta^{*} \in(0,1)$, and $\gamma^{*} \in\left(0,1-\delta^{*}\right)$ that satisfy condition (9). Since $G\left(r \mid z_{R}\right)=0$ requires $\gamma^{*}=\epsilon^{*}\left(1-\delta^{*}\right)$, where $\epsilon^{*} \in(0,1)$ is uniquely defined for each $\phi_{B} \in\left(\phi_{2}, \phi_{4}\right]$, we can rewrite (9) as:

$$
\frac{\phi_{B} \sum_{j=\frac{n+1}{2}}^{n-1}\left(\begin{array}{c}
n-1 \\
j
\end{array}\right)\left(1-\epsilon^{*}\left(1-\delta^{*}\right)\right)^{j}\left(\epsilon^{*}\left(1-\delta^{*}\right)\right)^{n-1-j}\left(1+\frac{1}{\epsilon^{*}} \phi_{B} \frac{\phi_{R}-1}{\phi_{B}-1}\right)^{n-1-j}}{\phi_{R}^{n-1} \sum_{j=\frac{n+1}{2}}^{n-1}\left(\begin{array}{c}
n-1 \\
j
\end{array}\right)\left(1-\epsilon^{*}\left(1-\delta^{*}\right)\right)^{j}\left(\epsilon^{*}\left(1-\delta^{*}\right)\right)^{n-1-j}\left(1+\frac{1}{\epsilon^{*}} \frac{\phi_{R}-1}{\phi_{R}\left(\phi_{B}-1\right)}\right)^{n-1-j}}=1
$$

Given $j-\frac{n-1}{2}=-\left(n-1-j-\frac{n-1}{2}\right)$, we can rearrange this condition by dividing both the numerator and the denominator by $\left(1-\epsilon^{*}\left(1-\delta^{*}\right)\right)^{\frac{n-1}{2}}\left(\epsilon^{*}\left(1-\delta^{*}\right)\right)^{\frac{n-1}{2}}$. This gives:

$$
\sum_{j=\frac{n+1}{2}}^{n-1}\left(\begin{array}{c}
n-1 \\
j
\end{array}\right)\left(\frac{1}{\epsilon^{*}\left(1-\delta^{*}\right)}-1\right)^{j-\frac{n-1}{2}}\left[\begin{array}{c}
\phi_{B}\left(1+\frac{1}{\epsilon^{*}} \phi_{B} \frac{\phi_{R}-1}{\phi_{B}-1}\right)^{n-1-j} \\
-\phi_{R}^{n-1}\left(1+\frac{1}{\epsilon^{*}} \frac{\phi_{R}-1}{\phi_{R}\left(\phi_{B}-1\right)}\right)^{n-1-j}
\end{array}\right]=0
$$


The first exponent is strictly positive when $j>\frac{n-1}{2}$, which is the case since the summation starts at $\frac{n+1}{2}$. Therefore the function is monotonic in $\delta^{*}$, which ensures single crossing and uniqueness over the domain.

The expression between square brackets in (10) is always negative for $j \geq \frac{n-1}{2}$. This comes from $G\left(r \mid z_{R}\right)=0, G\left(r \mid z_{R}\right)>G\left(r \mid z_{B}\right)$, and $\left.\phi_{B} \leq \phi_{4}\right)$. Since the LHS of equation (10) is the gain of playing $v$ (when $\delta^{*}<1$ ), we have that the gain of playing $v$ is strictly decreasing in $\delta$. We can also see that it is increasing in $\phi_{B}$, and equal to 0 in $\left\{\phi_{B}=\phi_{3}, \delta=0\right\}$. From part (v), we have that the gain of playing $v$ is also equal to 0 when $\left\{\phi_{B}=\phi_{4}, \delta=1\right\}$. Together, this implies that there exist a unique $\phi_{3}$ such that, for all $\phi_{3}<\phi_{B}<\phi_{4}$, there is a unique $0<\delta^{*}<1$ such that both $G\left(r \mid z_{R}\right)=0$ and $G\left(v \mid z_{B}\right)=0$.

$\operatorname{Part}(\mathbf{v}): \phi_{B} \in\left[\phi_{4}, \infty\right): \delta^{*}=1$ and $\gamma^{*}=\eta^{*}=\epsilon^{*}=0$.

For $\sigma_{r}\left(z_{R}\right)=1$ and $\sigma_{v}\left(z_{B}\right)=1$ to be an equilibrium, we need (v.a) $G\left(r \mid z_{R}\right) \geq 0$, (v.b) $G\left(r \mid z_{R}\right) \geq G\left(v \mid z_{R}\right)$, (v.c) $G\left(r \mid z_{B}\right) \leq G\left(v \mid z_{B}\right)$, and (v.d) $G\left(v \mid z_{B}\right) \geq 0$.

Note that under this strategy profile, we have $\tau_{b}^{\omega}=0, \forall \omega$. Hence, $\operatorname{Pr}\left(p i v_{R}^{\omega}\right)=0, \forall \omega$. First, this directly give $G\left(r \mid z_{R}\right)=0$, and condition (v.a) is satisfied. Second, condition (v.b) simplifies to $G\left(v \mid z_{R}\right) \leq 0$, which boils down to $\left(\phi_{R}\right)^{n} \geq 1$, which is always satisfied. Third, condition (v.c). becomes equivalent to condition (v.d): $G\left(v \mid z_{B}\right) \geq 0$, which boils down to $\phi_{B} \geq \phi_{4}$, which is satisfied in the present case.

\section{Appendix A2: Proofs of Section 4}

Proof of Theorem 1. We divide the proof into two parts. In part (i) we show that Veto weakly FIP dominates Unanimity and Majority for all values of the parameters. In part (ii) we show that Veto strictly dominates Unanimity unless $\phi_{R} \geq \phi_{4}$ and strictly dominates Majority unless $\beta=0$ and $\phi_{R} \leq \phi_{3}$.

\section{(i) Weak Full Information Pareto Dominance}

The strategy of this part of the proof is to look at the outcome that the three systems produce for all possible realized type profiles in the group of agents. We divide the proof in two parts: (i.a) there is at least one agent who has private values (i.e. $\exists i$ s.t. $z_{i}=z_{P}$ ), and (i.b) all agents have common values (i.e. $z_{i} \neq z_{P} \forall i$ ).

(i.a) $\exists i$ s.t. $z_{i}=z_{P}$. Since the private value agent votes $v$ under both $V$ and $U$, we have that these two systems implement the $D^{F I P C}$ with probability 1 . Under $M, v$ is not available. Therefore, $M$ does not implement the $D^{F I P C}$ with probability 1 . (It would require all voters to vote $b$ with probability 1 , but this is not an equilibrium strategy for common value agents).

(i.b) $z_{i} \neq z_{P} \forall i$.

First, notice that $\forall \mathbf{z}$ such that $z_{i} \neq z_{P} \forall i$, we have $D^{F I P C}\left(\mathbf{z}, \omega_{B}\right)=B$ and $D^{F I P C}\left(\mathbf{z}, \omega_{R}\right)=R$. Second, remember from Propositions 1 and 6 that under both $U$ and $V$ common-value agents behave as if $z_{i} \neq z_{P} \forall i$. This is not the case under $M$. Third, from McLennan (1998), we know that in pure common-value games, the strategy profile that maximizes the ex ante utility of the 
common value agents is an equilibrium. Fourth, in our model the disutility of both type of errors (i.e. $R$ in state $\omega_{B}$ and $B$ in state $\omega_{R}$ ) is identical for common value agents. Therefore, we have from McLennan (1998) that the strategy profile that maximize the probability that $R$ is chosen in state $\omega_{R}$ and $B$ is chosen in $\omega_{B}$ when $z_{n} \neq z_{P}$ must be an equilibrium. Fifth, notice that the action set in $U$ and $M$ is a strict subset of the action set under $V$. Therefore, any expected outcome under $U$ and $M$ can be reproduced under $V$. From McLennan (1998), this implies that the maximal equilibrium utility of common value agents under $V$ is greater than or equal to the maximal equilibrium utility of common value agents under $U$ and $M$.

\section{(ii) Strict Full Information Pareto Dominance}

We divide this part of the proof into four subparts to cover all the cases in which there is strict dominance.

(ii.a) Veto strictly FIP Dominates Majority when $\beta>0$. First, consider the realizations of the type profile for which there are no private value agents. In these cases, by the aforementioned McLennan (1998)'s logic, ex ante utility of the common value agents under $M$ can be at most as high as in the best equilibrium under $V$. But given $\beta>0$, with strictly positive probability there are realizations of the type profile with at least one private value agent. In those cases, $V$ implements the $D^{F I P C}$ with probability 1 while $M$ does it with a strictly lower probability. Hence, overall, $V$ strictly FIP Dominates $M$.

(ii.b) Veto strictly FIP Dominates Majority when $\beta=0$ and $\phi_{R}>\phi_{3}$. In those cases, there are only common value agents. For $\phi_{R}>\phi_{3}$, the equilibrium under $M$ cannot coincide with any of the equilibria under $V$ (as $v$ is played with positive probability). Therefore, by McLennan (1998), it must be that common value agents are better off under one of the equilibria under $V$ than under $M$.

(ii.c) Veto strictly FIP Dominates Unanimity when $\phi_{R}<\phi_{4}$. As is clear from part (i), $V$ and $U$ are equivalent in the presence of private value agents. Therefore, we need to show that $V$ strictly FIP Dominates $U$ for the realizations of the type profile for which there are only common value agents. Given that $V$ has two equilibria and that one reproduces the equilibrium under $U$, this can only happen if the ex ante utility of agents is strictly higher under the equilibrium characterized in Proposition 2 than in the equilibrium in Proposition 3. This is indeed the case, as will be shown in the proof of Theorem 2 .

Proof of Proposition 5. The proof consists of two parts, corresponding to cases (i) $\beta>0$ and (ii) $\beta=0$.

(i) If $\beta>0$, the probability that there is a private value agent as $n \longrightarrow \infty$ converges to one. Given that $\sigma_{v}^{*}\left(z_{P}\right)=1$, we have that Veto implements the FIP Criterion is equal to 1 .

(ii) If $\beta=0$, there are only common value agents. First, it is easy to see that $\phi_{3}, \phi_{4} \underset{n \rightarrow \infty}{\rightarrow} \infty$. Hence, for the equilibrium characterized in Proposition 2, we have that for any $\phi_{R}$ and $\phi_{B}$, in the limit $\sigma_{v}^{*}(z)=0 \forall z \in\left\{z_{R}, z_{B}\right\}$. The equilibrium with Veto will therefore coincide with the 
equilibrium under Majority. We know from Feddersen and Pesendorfer (1998) that, when $\beta=0$, Majority aggregates information perfectly in the limit. ${ }^{21}$ Hence, Veto aggregates information perfectly too.

\section{Proof of Theorem 2.}

For this proof, we assume that the equilibrium characterized in Proposition 2 is the relevant one under Veto. As already mentioned, the equilibrium of Proposition 3 is also the unique equilibrium under Unanimity (see the Supplementary Appendix for a full characterization). If agents play this equilibrium under Veto, then welfare is always identical under Veto and Unanimity.

First, note that both system deliver identical outcomes when $\exists i$ s.t. $z_{i}=z_{P}$ or $\phi_{B} \geq \phi_{4}$. All agents are then equally well off ex post in these cases. Hence, this also hold at the interim stage.

Now consider $z_{i} \neq z_{P} \forall i$ and $\phi_{B}<\phi_{4}$. The game is equivalent to the pure common-value game (i.e. $\beta=0$ ). Yet, even if all agents have the same preferences over outcomes, they have private information about the state of nature $\omega$. In that case, they may have different expected utility. We need to show that, for all $\phi_{R}, \phi_{B}$, and $n$, and for all possible realizations of $\mathbf{z}$ (conditional on $\left.z_{i} \neq z_{P} \forall i\right)$ the following condition holds:

$$
E_{\mathbf{z}, \omega}\left[u_{z}\left(D\left(\sigma^{* V}, \mathbf{z}\right), \omega\right)\right] \geq E_{\mathbf{z}, \omega}\left[u_{z}\left(D\left(\sigma^{* U}, \mathbf{z}\right), \omega\right)\right]
$$

for $z=z_{B}, z_{R}$, and with strict inequality for some $z$. We show that, except for $z_{R}$-agents when $\phi_{B} \in\left(\phi_{3}, \phi_{4}\right]$, condition (11) is always strictly satisfied. We split the proof in 5 steps.

Step 1: $z_{B}$-agents when $\phi_{B} \in\left[1, \phi_{4}\right]$.

Here, we show that a $z_{B}$-agent is strictly better off for $\phi_{B} \in\left[\phi_{1}, \phi_{3}\right]$, and equally well off for $\phi_{B} \in\left(\phi_{3}, \phi_{4}\right]$. First, see that the interim utility of a $z_{B}$ agent in that case is :

$$
\begin{aligned}
E_{\mathbf{z}, \omega}\left[u_{z_{B}}\left(D\left(\sigma^{* \Psi}, \mathbf{z}\right), \omega\right)\right] & =-\operatorname{Pr}\left(\omega_{R} \mid z_{B}\right) \operatorname{Pr}\left(B \mid \omega_{R}, \sigma^{* \Psi}\right)-\operatorname{Pr}\left(\omega_{B} \mid z_{B}\right) \operatorname{Pr}\left(R \mid \omega_{B}, \sigma^{* \Psi}\right) \\
& =-\frac{1}{1+\phi_{B}}+\frac{\operatorname{Pr}\left(R \mid \omega_{R}, \sigma^{* \Psi}\right)-\phi_{B} \operatorname{Pr}\left(R \mid \omega_{B}, \sigma^{* \Psi}\right)}{1+\phi_{B}}
\end{aligned}
$$

where $\operatorname{Pr}\left(D \mid \omega, \sigma^{* \Psi}\right)$ is the probability that $D$ is selected in state $\omega$ under voting system $\Psi$, for the associated equilibrium strategy profile $\sigma^{* \Psi}$.

Under $U$, the strategy of a $z_{B}$-agent is $\sigma_{r}^{* U}\left(z_{B}\right)=\alpha^{*}$, and $\sigma_{v}^{* U}\left(z_{B}\right)=1-\alpha^{*}$. If she plays $v$, then the decision is $B$ for sure, and her expected utility is minus the probability to be in state $\omega_{R}$ conditional on being type $z_{B}$, that is $-\frac{1}{1+\phi_{B}}$. As she is indifferent between playing $r$ and $v$, it must be that

$$
E_{\mathbf{z}, \omega}\left[u_{z_{B}}\left(D\left(\sigma^{* U}, \mathbf{z}\right), \omega\right)\right]=\frac{-1}{1+\phi_{B}}
$$

Under $V$, we consider the three parts in turn. First, when $\phi_{B} \in\left(\phi_{3}, \phi_{4}\right]$, the strategy of a $z_{B}$-agent is $\sigma_{b}^{* V}\left(z_{B}\right)=1-\delta^{*}$, and $\sigma_{v}^{* V}\left(z_{B}\right)=\delta^{*}$. Using the same logic as with unanimity, we

\footnotetext{
${ }^{21}$ Our setup is different than Feddersen and Pesendorfer (1998)'s. Yet, the proof, which is available upon request, is almost identical.
} 
have that in that case $z_{B}$-agent have the same expected utility:

$$
E_{\mathbf{z}, \omega}\left[u_{z_{B}}\left(D\left(\sigma^{* V}, \mathbf{z}\right), \omega\right)\right]=\frac{-1}{1+\phi_{B}}
$$

Second, when $\phi_{B} \in\left[\phi_{1}, \phi_{3}\right]$, a $z_{B}$-agent plays $b$ with probability 1 (that is, $\delta^{*}=0$ ). Hence, we have

$$
E_{\mathbf{z}, \omega}\left[u_{z_{B}}\left(D\left(\sigma^{* V}, \mathbf{z}\right), \omega\right)\right]=\frac{-1}{1+\phi_{B}}+\frac{\operatorname{Pr}\left(x_{r} \geq \frac{n+1}{2} \mid \omega_{R}, \sigma^{* V}\right)-\phi_{B} \operatorname{Pr}\left(x_{r} \geq \frac{n+1}{2} \mid \omega_{B}, \sigma^{* V}\right)}{1+\phi_{B}} .
$$

Given that $G^{V}\left(v \mid z_{B}\right)<0$, we have that condition (11) is strictly satisfied. Note the revealed preference argument: the agent could always ensure a level of expected utility equal to $\frac{-1}{1+\phi_{B}}$ by vetoing. If she does not do it, she will not be worse off as a result.

Third, when $\phi_{B} \in\left(1, \phi_{1}\right]$, the strategy of a $z_{B}$-agent is $\sigma_{r}^{* V}\left(z_{B}\right)=\eta^{*}$, and $\sigma_{b}^{* V}\left(z_{B}\right)=1-\eta^{*}$.

We thus have:

$$
\begin{aligned}
E_{\mathbf{z}, \omega}\left[u_{z_{B}}\left(D\left(\sigma^{* V}, \mathbf{z}\right), \omega\right)\right]= & \eta^{*}\left(\frac{-1}{1+\phi_{B}}+\frac{\operatorname{Pr}\left(x_{r} \geq \frac{n-1}{2} \mid \omega_{R}, \sigma^{* V}\right)-\phi_{B} \operatorname{Pr}\left(x_{r} \geq \frac{n-1}{2} \mid \omega_{B}, \sigma^{* V}\right)}{1+\phi_{B}}\right) \\
& +\left(1-\eta^{*}\right)\left(\frac{-1}{1+\phi_{B}}+\frac{\operatorname{Pr}\left(x_{r} \geq \frac{n+1}{2} \mid \omega_{R}, \sigma^{* V}\right)-\phi_{B} \operatorname{Pr}\left(x_{r} \geq \frac{n+1}{2} \mid \omega_{B}, \sigma^{* V}\right)}{1+\phi_{B}}\right) .
\end{aligned}
$$

Notice that

$$
\begin{aligned}
& \operatorname{Pr}\left(x_{r} \geq \frac{n+1}{2} \mid \omega_{R}, \sigma^{* V}\right)-\phi_{B} \operatorname{Pr}\left(x_{r} \geq \frac{n+1}{2} \mid \omega_{B}, \sigma^{* V}\right)=-G^{V}\left(v \mid z_{B}\right), \text { and } \\
& \operatorname{Pr}\left(x_{r} \geq \frac{n-1}{2} \mid \omega_{R}, \sigma^{* V}\right)-\phi_{B} \operatorname{Pr}\left(x_{r} \geq \frac{n-1}{2} \mid \omega_{B}, \sigma^{* V}\right)=-G^{V}\left(v \mid z_{B}\right)+G^{V}\left(r \mid z_{B}\right) .
\end{aligned}
$$

Given that $G^{V}\left(v \mid z_{B}\right)<0$ and that $G^{V}\left(r \mid z_{B}\right)=0$ in equilibrium, we have that

$$
E_{\mathbf{z}, \omega}\left[u_{z_{B}}\left(D\left(\sigma^{* V}, \mathbf{z}\right), \omega\right)\right]>\frac{-1}{1+\phi_{B}} .
$$

Hence, condition (11) is satisfied. The same revealed preference argument as above holds.

Step 2: $z_{R}$-agents when $\phi_{B} \in\left[\phi_{1}, \phi_{2}\right]$.

Under $U$, a $z_{R}$-agent always plays $r$, and her interim utility is (for all values of $\phi_{B}$ ):

$$
E_{\mathbf{z}, \omega}\left[u_{z_{R}}\left(D\left(\sigma^{* U}, \mathbf{z}\right), \omega\right)\right]=\frac{-\phi_{R}}{1+\phi_{R}}+\frac{\phi_{R} \operatorname{Pr}\left(x_{r}=n-1 \mid \omega_{R}, \sigma^{* U}\right)-\operatorname{Pr}\left(x_{r}=n-1 \mid \omega_{B}, \sigma^{* U}\right)}{1+\phi_{R}} .
$$

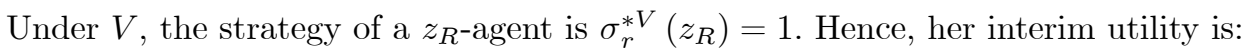

$$
E_{\mathbf{z}, \omega}\left[u_{z_{R}}\left(D\left(\sigma^{* V}, \mathbf{z}\right), \omega\right)\right]=\frac{-\phi_{R}}{1+\phi_{R}}+\frac{\phi_{R} \operatorname{Pr}\left(x_{r} \geq \frac{n-1}{2} \mid \omega_{R}, \sigma^{* V}\right)-\operatorname{Pr}\left(x_{r} \geq \frac{n-1}{2} \mid \omega_{B}, \sigma^{* V}\right)}{1+\phi_{R}} .
$$


For condition (11) to be satisfied, we need:

$$
\begin{aligned}
& \phi_{R} \operatorname{Pr}\left(x_{r} \geq \frac{n-1}{2} \mid \omega_{R}, \sigma^{* V}\right)-\operatorname{Pr}\left(x_{r} \geq \frac{n-1}{2} \mid \omega_{B}, \sigma^{* V}\right) \geq \\
& \phi_{R} \operatorname{Pr}\left(x_{r}=n-1 \mid \omega_{R}, \sigma^{* U}\right)-\operatorname{Pr}\left(x_{r}=n-1 \mid \omega_{B}, \sigma^{* U}\right),
\end{aligned}
$$

which becomes:

$$
\begin{gathered}
\sum_{j=\frac{n-1}{2}}^{n-1}\left(\begin{array}{c}
n-1 \\
j
\end{array}\right) \frac{\left[\phi_{R}\left(\phi_{R}\left(\phi_{B}-1\right)\right)^{j}\left(\phi_{R}-1\right)^{n-1-j}-\left(\phi_{B}-1\right)^{j}\left(\phi_{B}\left(\phi_{R}-1\right)\right)^{n-1-j}\right]}{\left(\phi_{B} \phi_{R}-1\right)^{n-1}} \geq \\
\frac{\phi_{R}\left(\phi_{R}\left(\phi_{B}-1\right)+\left(\phi_{R}-1\right) \alpha^{*}\right)^{n-1}-\left(\left(\phi_{B}-1\right)+\phi_{B}\left(\phi_{R}-1\right) \alpha^{*}\right)^{n-1}}{\left(\phi_{B} \phi_{R}-1\right)^{n-1}} .
\end{gathered}
$$

We can then rewrite the LHS as

$$
\frac{\left(\phi_{R}-1\right)^{n-1}}{\left(\phi_{B} \phi_{R}-1\right)^{n-1}} \sum_{j=\frac{n-1}{2}}^{n-1}\left(\begin{array}{c}
n-1 \\
j
\end{array}\right)\left(\frac{\phi_{B}-1}{\phi_{R}-1}\right)^{j}\left[\left(\phi_{R}\right)^{j+1}-\left(\phi_{B}\right)^{n-1-j}\right] .
$$

We can also distribute the RHS, and group it similarly as the LHS:

$$
\frac{\left(\phi_{R}-1\right)^{n-1}}{\left(\phi_{B} \phi_{R}-1\right)^{n-1}} \sum_{0}^{n-1}\left(\begin{array}{c}
n-1 \\
j
\end{array}\right)\left(\frac{\phi_{B}-1}{\phi_{R}-1}\right)^{j}\left(\alpha^{*}\right)^{n-1-j}\left[\left(\phi_{R}\right)^{j+1}-\left(\phi_{B}\right)^{n-1-j}\right] \text {. }
$$

We therefore need to show:

$$
\begin{gathered}
\sum_{j=\frac{n-1}{2}}^{n-1}\left(\begin{array}{c}
n-1 \\
j
\end{array}\right)\left(\frac{\phi_{B}-1}{\phi_{R}-1}\right)^{j}\left[\left(\phi_{R}\right)^{j+1}-\left(\phi_{B}\right)^{n-1-j}\right]> \\
\sum_{0}^{n-1}\left(\begin{array}{c}
n-1 \\
j
\end{array}\right)\left(\frac{\phi_{B}-1}{\phi_{R}-1}\right)^{j}\left(\alpha^{*}\right)^{n-1-j}\left[\left(\phi_{R}\right)^{j+1}-\left(\phi_{B}\right)^{n-1-j}\right]
\end{gathered}
$$

We can now compare these two sums term by term (recall that here $\phi_{B} \in\left[\phi_{1}, \phi_{2}\right]$ ) and show that the LHS terms are always larger that the RHS terms. First, observe that the terms in $j=n-1$ cancel out. Second, for $j \in\left[\frac{n-1}{2}, n-1\right)$, note that from $\phi_{B} \leq \phi_{2} \equiv\left(\phi_{R}\right)^{\frac{n+1}{n-1}}$, we have that

$$
\left(\phi_{R}\right)^{j+1} \geq\left(\phi_{B}\right)^{n-1-j}
$$

with strict inequality for $j>\frac{n-1}{2}$. Since $\alpha^{*}<1$, the LHS terms are strictly higher than the RHS terms $\forall j \in\left[\frac{n-1}{2}, n-1\right)$. Third, for $j<\frac{n-1}{2}$, note that from $\phi_{B} \geq \phi_{1} \equiv\left(\phi_{R}\right)^{\frac{n-1}{n+1}}$, we have that

$$
\left(\phi_{R}\right)^{j+1} \leq\left(\phi_{B}\right)^{n-1-j}
$$

with strict inequality for $j<\frac{n-3}{2}$. So, all the respective terms on the RHS are negative and they are equal to 0 in the LHS.

Step 3: $z_{R}$-agents when $\phi_{B} \in\left(\phi_{2}, \phi_{3}\right]$.

Under $U$, the interim utility of a $z_{R}$-agent is still given by (12). Under $V$, the strategy of a 


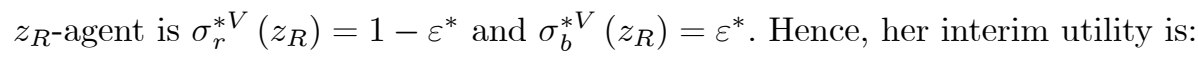

$$
\begin{aligned}
E_{\mathbf{z}, \omega}\left[u_{z_{R}}\left(D\left(\sigma^{* V}, \mathbf{z}\right), \omega\right)\right]= & \left(1-\varepsilon^{*}\right)\left(\frac{-\phi_{R}}{1+\phi_{R}}+\frac{\phi_{R} \operatorname{Pr}\left(x_{r} \geq \frac{n-1}{2} \mid \omega_{R}, \sigma^{* V}\right)-\operatorname{Pr}\left(x_{r} \geq \frac{n-1}{2} \mid \omega_{B}, \sigma^{* V}\right)}{1+\phi_{R}}\right) \\
& +\varepsilon^{*}\left(\frac{-\phi_{R}}{1+\phi_{R}}+\frac{\phi_{R} \operatorname{Pr}\left(x_{r} \geq \frac{n+1}{2} \mid \omega_{R}, \sigma^{* V}\right)-\operatorname{Pr}\left(x_{r} \geq \frac{n+1}{2} \mid \omega_{B}, \sigma^{* V}\right)}{1+\phi_{R}}\right) .
\end{aligned}
$$

Since $z_{R}$-agents are randomizing, the two parentheses must be equal in equilibrium (which requires $\left.\phi_{R} \operatorname{Pr}\left(x_{r}=\frac{n-1}{2} \mid \omega_{R}, \sigma^{* V}\right)=\operatorname{Pr}\left(x_{r}=\frac{n-1}{2} \mid \omega_{B}, \sigma^{* V}\right)\right)$. Therefore, condition (11) is satisfied iff

$\phi_{R} \operatorname{Pr}\left(x_{r} \geq \frac{n+1}{2} \mid \omega_{R}, \sigma^{* V}\right)-\operatorname{Pr}\left(x_{r} \geq \frac{n+1}{2} \mid \omega_{B}, \sigma^{* V}\right) \geq \phi_{R} \operatorname{Pr}\left(x_{r}=n-1 \mid \omega_{R}, \sigma^{* U}\right)-\operatorname{Pr}\left(x_{r}=n-1 \mid \omega_{B}, \sigma^{* U}\right)$.

Note that

$$
\begin{aligned}
& \left(\phi_{R} \phi_{B}-1\right)^{n-1}\left(\phi_{R} \operatorname{Pr}\left(x_{r} \geq \frac{n+1}{2} \mid \omega_{R}, \sigma^{* V}\right)-\operatorname{Pr}\left(x_{r} \geq \frac{n+1}{2} \mid \omega_{B}, \sigma^{* V}\right)\right)= \\
& \sum_{j=\frac{n+1}{2}}^{n-1}\left(\begin{array}{c}
n-1 \\
j
\end{array}\right)\left(1-\varepsilon^{*}\right)^{j}\left(\phi_{B}-1\right)^{j}\left[\begin{array}{c}
\left(\phi_{R}\right)^{j+1}\left(\varepsilon^{*} \phi_{R}\left(\phi_{B}-1\right)+\left(\phi_{R}-1\right)\right)^{n-1-j} \\
-\left(\varepsilon^{*}\left(\phi_{B}-1\right)+\phi_{B}\left(\phi_{R}-1\right)\right)^{n-1-j}
\end{array}\right] .
\end{aligned}
$$

We know that the term in $j=\frac{n-1}{2}$ is nil (because it corresponds to $G^{V}\left(r \mid z_{R}\right.$ ), which is nil at this equilibrium) and it is trivial to show that the equivalent terms in $j<\frac{n-1}{2}$ are strictly negative. Therefore, we have that:

$$
\begin{aligned}
& \left(\phi_{R} \phi_{B}-1\right)^{n-1}\left(\phi_{R} \operatorname{Pr}\left(x_{r} \geq \frac{n+1}{2} \mid \omega_{R}, \sigma^{* V}\right)-\operatorname{Pr}\left(x_{r} \geq \frac{n+1}{2} \mid \omega_{B}, \sigma^{* V}\right)\right)> \\
& \sum_{j=0}^{n-1}\left(\begin{array}{c}
n-1 \\
j
\end{array}\right)\left(\left(1-\varepsilon^{*}\right)\left(\phi_{B}-1\right)\right)^{j}\left[\begin{array}{c}
\left(\phi_{R}\right)^{j+1}\left(\varepsilon^{*} \phi_{R}\left(\phi_{B}-1\right)+\left(\phi_{R}-1\right)\right)^{n-1-j} \\
-\left(\varepsilon^{*}\left(\phi_{B}-1\right)+\phi_{B}\left(\phi_{R}-1\right)\right)^{n-1-j}
\end{array}\right] .
\end{aligned}
$$

Using the Binomial Theorem, this can be rewritten as

$$
\begin{aligned}
& \left(\phi_{R} \phi_{B}-1\right)^{n-1}\left(\phi_{R} \operatorname{Pr}\left(x_{r} \geq \frac{n+1}{2} \mid \omega_{R}, \sigma^{* V}\right)-\operatorname{Pr}\left(x_{r} \geq \frac{n+1}{2} \mid \omega_{B}, \sigma^{* V}\right)\right) \\
> & \phi_{R}\left[\phi_{R}\left(\phi_{B}-1\right)+\left(\phi_{R}-1\right)\right]^{n-1}-\left[\left(\phi_{B}-1\right)+\phi_{B}\left(\phi_{R}-1\right)\right]^{n-1} .
\end{aligned}
$$

Therefore, condition (11) is satisfied if

$$
\begin{aligned}
& \phi_{R}\left[\phi_{R}\left(\phi_{B}-1\right)+\left(\phi_{R}-1\right)\right]^{n-1}-\left[\left(\phi_{B}-1\right)+\phi_{B}\left(\phi_{R}-1\right)\right]^{n-1} \\
\geq & \phi_{R}\left[\phi_{R}\left(\phi_{B}-1\right)+\left(\phi_{R}-1\right) \alpha^{*}\right]^{n-1}-\left(\left(\phi_{B}-1\right)+\phi_{B}\left(\phi_{R}-1\right) \alpha^{*}\right)^{n-1} .
\end{aligned}
$$

To prove that this is always satisfied, we derive the RHS with respect to $\alpha^{*}$ and use the fact that $G^{U}\left(v \mid z_{B}\right)=0$ in equilibrium requires

$$
\left[\phi_{R}\left(\phi_{B}-1\right)+\left(\phi_{R}-1\right) \alpha^{*}\right]=\phi_{B}^{\frac{1}{n-1}}\left[\left(\phi_{B}-1\right)+\phi_{B}\left(\phi_{R}-1\right) \alpha^{*}\right]
$$


First, the derivative of the RHS with respect to $\alpha^{*}$ is

$$
(n-1)\left(\phi_{R}-1\right)\left[\phi_{R}\left[\phi_{R}\left(\phi_{B}-1\right)+\left(\phi_{R}-1\right) \alpha^{*}\right]^{n-2}-\phi_{B}\left(\left(\phi_{B}-1\right)+\phi_{B}\left(\phi_{R}-1\right) \alpha^{*}\right)^{n-2}\right]>0 .
$$

Substituting for $G^{U}\left(v \mid z_{B}\right)=0$, this condition boils down to

$$
\phi_{R} \phi_{B}^{\frac{-1}{n-1}}>1
$$

which is always strictly satisfied when $\phi_{B}<\phi_{4}$.

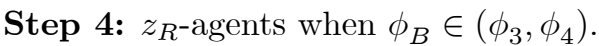

Recall that the strategy that maximizes ex ante welfare in a common value game must be an equilibrium (McLennan, 1998). There are two possible candidates: the equilibria described in Proposition 2 and the one in Proposition 3, which reproduces the equilibrium with unanimity. We first show that the equilibrium characterized in Proposition 2 strictly ex ante dominates Unanimity. We do this by constructing a (non-equilibrium) strategy profile that implies higher interim utility for both agents that equilibrium that under the Proposition 3 equilibrium.

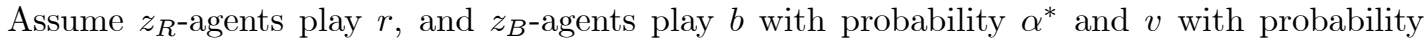
$1-\alpha^{*}$, where $\alpha^{*}$ is the equilibrium probability with which they play $r$ at the Proposition 3 equilibrium.

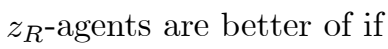

$$
\begin{gathered}
\left.\sum_{j=\frac{n-1}{2}}^{n-1} \begin{array}{c}
n-1 \\
j
\end{array}\right) \frac{\left[\phi_{R}\left(\phi_{R}\left(\phi_{B}-1\right)\right)^{j}\left(\alpha^{*}\left(\phi_{R}-1\right)\right)^{n-1-j}-\left(\phi_{B}-1\right)^{j}\left(\alpha^{*} \phi_{B}\left(\phi_{R}-1\right)\right)^{n-1-j}\right]}{\left(\phi_{B} \phi_{R}-1\right)^{n-1}} \geq \\
\frac{\phi_{R}\left(\phi_{R}\left(\phi_{B}-1\right)+\left(\phi_{R}-1\right) \alpha^{*}\right)^{n-1}-\left(\left(\phi_{B}-1\right)+\phi_{B}\left(\phi_{R}-1\right) \alpha^{*}\right)^{n-1}}{\left(\phi_{B} \phi_{R}-1\right)^{n-1}} .
\end{gathered}
$$

We can then rewrite the LHS as

$$
\frac{\left(\phi_{R}-1\right)^{n-1}}{\left(\phi_{B} \phi_{R}-1\right)^{n-1}} \sum_{j=\frac{n-1}{2}}^{n-1}\left(\begin{array}{c}
n-1 \\
j
\end{array}\right)\left(\frac{\phi_{B}-1}{\phi_{R}-1}\right)^{j}\left(\alpha^{*}\right)^{n-1-j}\left[\left(\phi_{R}\right)^{j+1}-\left(\phi_{B}\right)^{n-1-j}\right] .
$$

We can also distribute the RHS, and group it similarly as the LHS:

$$
\frac{\left(\phi_{R}-1\right)^{n-1}}{\left(\phi_{B} \phi_{R}-1\right)^{n-1}} \sum_{0}^{n-1}\left(\begin{array}{c}
n-1 \\
j
\end{array}\right)\left(\frac{\phi_{B}-1}{\phi_{R}-1}\right)^{j}\left(\alpha^{*}\right)^{n-1-j}\left[\left(\phi_{R}\right)^{j+1}-\left(\phi_{B}\right)^{n-1-j}\right] .
$$

And it is obvious that the condition holds strictly since we have that $\left(\phi_{R}\right)^{j+1} \leq\left(\phi_{B}\right)^{n-1-j}$ for all $j \leq \frac{n-3}{2}$ since $\phi_{B} \geq \phi_{3} \equiv\left(\phi_{R}\right)^{\frac{n+1}{n-1}}$.

$z_{B}$-agents are better off if

$$
\begin{gathered}
\sum_{j=\frac{n-1}{2}}^{n-1}\left(\begin{array}{c}
n-1 \\
j
\end{array}\right) \frac{\left[\left(\phi_{R}\left(\phi_{B}-1\right)\right)^{j}\left(\alpha^{*}\left(\phi_{R}-1\right)\right)^{n-1-j}-\phi_{B}\left(\phi_{B}-1\right)^{j}\left(\alpha^{*} \phi_{B}\left(\phi_{R}-1\right)\right)^{n-1-j}\right]}{\left(\phi_{B} \phi_{R}-1\right)^{n-1}} \geq \\
\frac{\left(\phi_{R}\left(\phi_{B}-1\right)+\left(\phi_{R}-1\right) \alpha^{*}\right)^{n-1}-\phi_{B}\left(\left(\phi_{B}-1\right)+\phi_{B}\left(\phi_{R}-1\right) \alpha^{*}\right)^{n-1}}{\left(\phi_{B} \phi_{R}-1\right)^{n-1}} .
\end{gathered}
$$


We can then rewrite the LHS as

$$
\frac{\left(\phi_{R}-1\right)^{n-1}}{\left(\phi_{B} \phi_{R}-1\right)^{n-1}} \sum_{j=\frac{n+1}{2}}^{n-1}\left(\begin{array}{c}
n-1 \\
j
\end{array}\right)\left(\frac{\phi_{B}-1}{\phi_{R}-1}\right)^{j}\left(\alpha^{*}\right)^{n-1-j}\left[\left(\phi_{R}\right)^{j}-\left(\phi_{B}\right)^{n-j}\right] .
$$

We can also distribute the RHS, and group it similarly as the LHS:

$$
\frac{\left(\phi_{R}-1\right)^{n-1}}{\left(\phi_{B} \phi_{R}-1\right)^{n-1}} \sum_{0}^{n-1}\left(\begin{array}{c}
n-1 \\
j
\end{array}\right)\left(\frac{\phi_{B}-1}{\phi_{R}-1}\right)^{j}\left(\alpha^{*}\right)^{n-1-j}\left[\left(\phi_{R}\right)^{j}-\left(\phi_{B}\right)^{n-j}\right] .
$$

And it is obvious that the condition holds since we have that: $\left(\phi_{R}\right)^{j} \leq\left(\phi_{B}\right)^{n-j}$ for all $j \leq \frac{n-1}{2}$ since $\phi_{B} \geq \phi_{3} \equiv\left(\phi_{R}\right)^{\frac{n+1}{n-1}}$.

Hence, it must be that Veto strictly ex ante dominates Unanimity when $\phi_{B} \in\left(\phi_{3}, \phi_{4}\right)$. Finally, since $z_{B}$-agents are equally well off under Veto (Step 1 ) and Unanimity, it must be that $z_{R^{-a g e n t s}}$ are strictly better off.

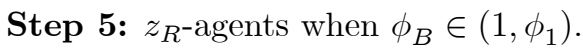

First we use $\eta^{*}$ and $\alpha^{*}$ (from Propositions 2 and 7) to derive the probabilities that the reform is adopted (conditional on the agent being $z_{R}$ ) in each state under both rules (remember that $\sigma_{r}^{* V}\left(z_{R}\right)=1=\sigma_{r}^{* U}\left(z_{R}\right)$ in the case under consideration). Denoting by $\Omega^{\Psi}(\omega)$ the probability that the reform is adopted in state $\omega$ under voting system $\Psi$, we have

$$
\begin{aligned}
\Omega^{V}\left(\omega_{R}\right) & \equiv \operatorname{Pr}\left(x_{r} \geq \frac{n-1}{2} \text { and } x_{v}=0 \mid \omega_{R}, \sigma^{* V}\right) \\
& =\frac{1}{\left(\left(\phi_{B}\right)^{\frac{2 n}{n-1}}-1\right)^{n-1}} \sum_{j=\frac{n-1}{2}}^{n-1}\left(\begin{array}{c}
n-1 \\
j
\end{array}\right)\left(\phi_{B}\right)^{j \frac{n+1}{n-1}}\left[\left(\phi_{B}-1\right)^{j}\left(\left(\phi_{B}\right)^{\frac{n+1}{n-1}}-1\right)^{n-1-j}\right] \\
\Omega^{V}\left(\omega_{B}\right) & \equiv \operatorname{Pr}\left(x_{r} \geq \frac{n-1}{2} \text { and } x_{v}=0 \mid \omega_{B}, \sigma^{* V}\right) \\
& =\frac{1}{\left(\left(\phi_{B}\right)^{\frac{2 n}{n-1}}-1\right)^{n-1}} \sum_{j=\frac{n-1}{2}}^{n-1}\left(\begin{array}{c}
n-1 \\
j
\end{array}\right)\left(\phi_{B}\right)^{n-1-j}\left[\left(\phi_{B}-1\right)^{j}\left(\left(\phi_{B}\right)^{\frac{n+1}{n-1}}-1\right)^{n-1-j}\right] \\
\Omega^{U}\left(\omega_{R}\right) & \equiv \operatorname{Pr}\left(x_{r}=n-1 \mid \omega_{R}, \sigma^{* U}\right)=\phi_{B} \frac{\left(\phi_{B}-1\right)^{n-1}}{\left(\left(\phi_{B}\right)^{\frac{n}{n-1}}-1\right)^{n-1}}, \text { and } \\
\Omega^{U}\left(\omega_{B}\right) & \equiv \operatorname{Pr}\left(x_{r}=n-1 \mid \omega_{B}, \sigma^{* U}\right)=\frac{\left(\phi_{B}-1\right)^{n-1}}{\left(\left(\phi_{B}\right)^{\frac{n}{n-1}}-1\right)^{n-1}} .
\end{aligned}
$$

Note that they are all independent from $\phi_{R}$.

The interim utility of a $z_{R}$-agent is given by (13) and (12) under Veto and Unanimity, respectively. Therefore, $E_{\mathbf{z}, \omega}\left[u_{z_{R}}\left(D\left(\sigma^{* V}, \mathbf{z}\right), \omega\right)\right]-E_{\mathbf{z}, \omega}\left[u_{z_{R}}\left(D\left(\sigma^{* U}, \mathbf{z}\right), \omega\right)\right]$ is given by

$$
\frac{\phi_{R} \Omega^{V}\left(\omega_{R}\right)-\Omega^{V}\left(\omega_{B}\right)}{1+\phi_{R}}-\frac{\phi_{R} \Omega^{U}\left(\omega_{R}\right)-\Omega^{U}\left(\omega_{B}\right)}{1+\phi_{R}}
$$

From step 2, we know that $E_{\mathbf{z}, \omega}\left[u_{z_{R}}\left(D\left(\sigma^{* V}, \mathbf{z}\right), \omega\right)\right]>E_{\mathbf{z}, \omega}\left[u_{z_{R}}\left(D\left(\sigma^{* U}, \mathbf{z}\right), \omega\right)\right]$ when $\phi_{B}=\phi_{1}$. 
To prove that this inequality holds for any $\phi_{B} \in\left(1, \phi_{R}\right)$, it is sufficient to prove that it is satisfied for any $\phi_{R}$ larger than the $\phi_{R}$ such that $\phi_{B}=\phi_{1}$. Given that $\Omega^{\Psi}(\omega)$ is independent of $\phi_{R}$ for all $\Psi$ and $\omega$, a sufficient condition for $E_{\mathbf{z}, \omega}\left[u_{z_{R}}\left(D\left(\sigma^{* V}, \mathbf{z}\right), \omega\right)\right]>E_{\mathbf{z}, \omega}\left[u_{z_{R}}\left(D\left(\sigma^{* U}, \mathbf{z}\right), \omega\right)\right]$ to be satisfied for larger $\phi_{R}$ is that $\Omega^{V}\left(\omega_{R}\right) \geq \Omega^{U}\left(\omega_{R}\right)$. Hence, we need to prove that the following inequality is satisfied when $\phi_{B} \in\left(1, \phi_{1}\right)$ :

$$
\sum_{j=\frac{n-1}{2}}^{n-1}\left(\begin{array}{c}
n-1 \\
j
\end{array}\right) \frac{\left[\left(\phi_{B}-1\right) \phi_{B}^{\frac{n+1}{n-1}}\right]^{j}\left[\left(\phi_{B}^{\frac{n+1}{n-1}}-1\right)\right]^{n-1-j}}{\left(\phi_{B}^{\frac{2 n}{n-1}}-1\right)^{n-1}}>\phi_{B} \frac{\left(\phi_{B}-1\right)^{n-1}}{\left(\left(\phi_{B}\right)^{\frac{n}{n-1}}-1\right)^{n-1}} .
$$

This boils down to

$$
\sum_{j=\frac{n-1}{2}}^{n-1}\left(\begin{array}{c}
n-1 \\
j
\end{array}\right)\left[\left(\phi_{B}-1\right) \phi_{B}^{\frac{n+1}{n-1}}\right]^{j}\left[\left(\phi_{B}^{\frac{n+1}{n-1}}-1\right)\right]^{n-1-j}>\phi_{B}\left(\left(\phi_{B}\right)^{\frac{n}{n-1}}+1\right)^{n-1}\left(\phi_{B}-1\right)^{n-1}
$$

Using Lemma 1 (in this Appendix), we can substitute for $\left(\phi_{B}^{\frac{n+1}{n-1}}-1\right)$ and cancel the terms in $\left(\phi_{B}-1\right)^{n-1}$. This gives:

$$
\sum_{j=\frac{n-1}{2}}^{n-1}\left(\begin{array}{c}
n-1 \\
j
\end{array}\right) \phi_{B}^{j \frac{n+1}{n-1}}\left[\left(\frac{n+1}{n-1} \phi_{B}^{\frac{1}{n-1}}\right]^{n-1-j}>\phi_{B}\left(\left(\phi_{B}\right)^{\frac{n}{n-1}}+1\right)^{n-1}\right.
$$

Using the Binomial Theorem, we have

$$
\sum_{j=\frac{n-1}{2}}^{n-1}\left(\begin{array}{c}
n-1 \\
j
\end{array}\right) \phi_{B}^{j \frac{n}{n-1}}\left[\frac{n+1}{n-1}\right]^{n-1-j}>\sum_{k=0}^{n-1}\left(\begin{array}{c}
n-1 \\
k
\end{array}\right)\left(\phi_{B}\right)^{k \frac{n}{n-1}}
$$

Given that the terms in $j=n-1=k$ cancel out, we can focus on $j<n-1$ and $k<n-1$. Note that, for all $j<n-1$

$$
\left[\frac{n+1}{n-1}\right]^{n-1-j}=\left[1+\frac{2}{n-1}\right]^{n-1-j}=\left[1+\frac{\frac{2(n-1-j)}{n-1}}{n-1-j}\right]^{n-1-j} \geq 1+\frac{n-1-j}{\frac{n-1}{2}}
$$

(Indeed, for $x \geq-1$ and $r \in \mathbb{R} \backslash(0,1)$, we know that $(1+x)^{r} \geq 1+r x$ is satisfied). Thus, it is sufficient to show that:

$$
\sum_{j=\frac{n-1}{2}}^{n-2}\left(\begin{array}{c}
n-1 \\
j
\end{array}\right) \phi_{B}^{j \frac{n}{n-1}}\left(1+\frac{n-1-j}{\frac{n-1}{2}}\right)>\sum_{k=0}^{n-2}\left(\begin{array}{c}
n-1 \\
k
\end{array}\right)\left(\phi_{B}\right)^{k \frac{n}{n-1}}
$$

or

$$
\sum_{j=\frac{n-1}{2}}^{n-2} \frac{(n-1) !}{(n-1-j) ! j !}\left(\frac{n-1-j}{\frac{n-1}{2}}\right)\left(\phi_{B}\right)^{j \frac{n}{n-1}}>\sum_{k=0}^{\frac{n-3}{2}} \frac{(n-1) !}{(n-1-k) ! k !}\left(\phi_{B}\right)^{k \frac{n}{n-1}} .
$$

Let us compare the terms two-by-two in the following order: $j=\frac{n-1}{2}$ with $k=\frac{n-3}{2}, j=\frac{n+1}{2}$ with $k=\frac{n-5}{2}, j=\frac{n+3}{2}$ with $k=\frac{n-7}{2}, \ldots$ and $j=n-2$ with $k=0$. This comparison boils down 
to:

$$
\frac{n-1-j}{\frac{n-1}{2}} \frac{(n-1) !}{(n-1-j) ! j !} \geq \frac{(n-1) !}{(j+1) !(n-1-j-1) !} .
$$

Simple algebra gives $j \geq \frac{n-1}{2}-1$, which is satisfied since we consider all $j \in\left\{\frac{n-1}{2}, \frac{n+1}{2}, \ldots, n-2\right\}$.

Lemma 1 For all $x \geq 1, n>1$, we have that

$$
\left(x^{\frac{n+1}{n-1}}-1\right) \geq \frac{n+1}{n-1}\left(x^{\frac{n}{n-1}}-x^{\frac{1}{n-1}}\right) .
$$

Proof. Let us denote the $\left(x^{\frac{n+1}{n-1}}-1\right)$ by $f(x)$, and $\frac{n+1}{n-1}\left(x^{\frac{n}{n-1}}-x^{\frac{1}{n-1}}\right)$ by $g(x)$.

Note that (i) $f(1)=g(1)=0$, and (ii) $f(x)>0, g(x)>0$ for all $x>1$, and $n>1$. Therefore, it is sufficient to prove that $f^{\prime}(x) \geq g^{\prime}(x)$ for all $x \geq 1$, and $n>1$. Taking the first derivatives, we obtain

$$
\begin{aligned}
& f^{\prime}(x)=\frac{n+1}{n-1} x^{\frac{2}{n-1}}, \text { and } \\
& g^{\prime}(x)=\frac{n+1}{n-1}\left[\frac{n}{n-1} x^{\frac{1}{n-1}}-\frac{1}{n-1} x^{\frac{1}{n-1}-1}\right] .
\end{aligned}
$$

Thus, we have that $f^{\prime}(x) \geq g^{\prime}(x)$ if

$$
\frac{x^{\frac{2}{n-1}}}{x^{\frac{1}{n-1}}} \geq \frac{n}{n-1} \frac{x^{\frac{1}{n-1}}}{x^{\frac{1}{n-1}}}-\frac{1}{n-1} \frac{x^{\frac{1}{n-1}-1}}{x^{\frac{1}{n-1}}}
$$

which simplifies to $(n-1) x^{\frac{1}{n-1}} \geq n-x^{-1}$. Denoting $(n-1) x^{\frac{1}{n-1}}$ by $h(x)$ and $\left(n-x^{-1}\right)$ by $k(x)$, we have that

$$
\begin{aligned}
h^{\prime}(x) & =x^{\frac{1}{n-1}-1}, \text { and } \\
k^{\prime}(x) & =x^{-2} .
\end{aligned}
$$

Therefore, for all $x>1, n>1$, we have $h^{\prime}(x)>k^{\prime}(x)$. Given that $h(1)=k(1)=n-1$, we have that, for all $x>1, n>1, h(x)>k(x)$ and thus that $f(x) \geq g(x)$.

\section{Supplementary Appendix (not for publication)}

\section{Threshold for equilibrium behavior}

We define the four thresholds $\phi_{1}, \phi_{2}, \phi_{3}$ and $\phi_{4}$ (as functions of $\phi_{R}$ and $n$ ) for the values of $\phi_{B}$ at which equilibrium behavior "changes", and prove that $1<\phi_{1}<\phi_{2} \leq \phi_{3} \leq \phi_{4}<\infty$.

Each threshold corresponds to a set of equilibrium conditions: $\phi_{1}$ corresponds to $G\left(r \mid z_{B}\right)=0$ when $\sigma_{r}\left(z_{R}\right)=1$ and $\sigma_{b}\left(z_{B}\right)=1, \phi_{2}$ corresponds to $G\left(r \mid z_{R}\right)=0$ when $\sigma_{r}\left(z_{R}\right)=1$ and $\sigma_{b}\left(z_{B}\right)=$ 
1, $\phi_{4}$ corresponds to $G\left(r \mid z_{R}\right)=0$ when $\sigma_{r}\left(z_{R}\right)=1$ and $\sigma_{v}\left(z_{B}\right)=1$. These three thresholds can be solved for in closed form:

$$
\phi_{1}=\left(\phi_{R}\right)^{\frac{n-1}{n+1}}, \phi_{2}=\left(\phi_{R}\right)^{\frac{n+1}{n-1}}, \phi_{4}=\left(\phi_{R}\right)^{n-1}
$$

and it is straightforward to see that for all couples $\left(n, \phi_{R}\right)$, we have $1<\phi_{1}<\phi_{2} \leq \phi_{4}<\infty$.

The remaining threshold $\phi_{3}$ corresponds to $G\left(v \mid z_{B}\right)=0$ when $\sigma_{b}\left(z_{R}\right)=\epsilon^{*}$, and $\sigma_{b}\left(z_{B}\right)=1 .^{22}$ It is implicitly defined by:

$$
\phi_{3}=\phi_{R}^{n-1} \frac{\sum_{j=\frac{n+1}{2}}^{n-1}\left(\begin{array}{c}
n-1 \\
j
\end{array}\right)\left(1-\epsilon^{*}\right)^{j}\left(\phi_{3}-1\right)^{j}\left(\epsilon^{*}\left(\phi_{3}-1\right)+\frac{\left(\phi_{R}-1\right)}{\phi_{R}}\right)^{n-1-j}}{\sum_{j=\frac{n+1}{2}}^{n-1}\left(\begin{array}{c}
n-1 \\
j
\end{array}\right)\left(1-\epsilon^{*}\right)^{j}\left(\phi_{3}-1\right)^{j}\left(\epsilon^{*}\left(\phi_{3}-1\right)+\phi_{3}\left(\phi_{R}-1\right)\right)^{n-1-j}}
$$

where $\epsilon^{*}=\bar{\epsilon}$, if $\bar{\epsilon}=\frac{\phi_{3}\left(1-\phi_{R}\right)-\left(\phi_{R}\right)^{\frac{n+1}{n-1}}\left(1-\phi_{R}\right)}{\phi_{R}\left(1-\phi_{3}\right)-\phi_{R}^{-\frac{n+1}{n-1}}\left(1-\phi_{3}\right)} \in[0,1]$ and $\epsilon^{*}=0$ otherwise. We need to show that $\phi_{2} \leq \phi_{3} \leq \phi_{4}$.

First, note that when $n=3$, this expression simplifies to $\phi_{3}=\phi_{R}^{n-1}$, and $\phi_{2}=\phi_{3}=\phi_{4}$. To study the case where $n>3$, we construct the following function of $\phi$, of which $\phi_{3}$ is a zero:

$$
g(\phi)=\phi-\phi_{R}^{n-1} \frac{\sum_{j=\frac{n+1}{2}}^{n-1}\left(\begin{array}{c}
n-1 \\
j
\end{array}\right)\left(1-\epsilon^{*}\right)^{j}(\phi-1)^{j}\left(\epsilon^{*}(\phi-1)+\frac{\left(\phi_{R}-1\right)}{\phi_{R}}\right)^{n-1-j}}{\sum_{j=\frac{n+1}{2}}^{n-1}\left(\begin{array}{c}
n-1 \\
j
\end{array}\right)\left(1-\epsilon^{*}\right)^{j}(\phi-1)^{j}\left(\epsilon^{*}(\phi-1)+\phi\left(\phi_{R}-1\right)\right)^{n-1-j}}
$$

And we show that this function is (i) strictly positive above $\phi_{4}$; (ii) strictly negative below $\phi_{2}$; from which we conclude that it has a zero between $\phi_{2}$ and $\phi_{4}$. First, $g(\phi)>0$ for all $\phi \geq \phi_{4}=\phi_{R}^{n-1}$ because the denominator in the RHS of (15) is always strictly larger than the numerator when $n>3$. Second, to see that $g(\phi)<0$ for all $\phi \leq \phi_{2}$, note that when $\phi \leq \phi_{2}$ then $\epsilon^{*}=0$. Hence, $g(\phi)<0$ simplifies to:

$$
\sum_{j=\frac{n+1}{2}}^{n-1}\left(\begin{array}{c}
n-1 \\
j
\end{array}\right)(\phi-1)^{j}\left(\phi\left(\phi_{R}-1\right)\right)^{n-1-j}<\phi_{R}^{n-1} \sum_{j=\frac{n+1}{2}}^{n-1}\left(\begin{array}{c}
n-1 \\
j
\end{array}\right)(\phi-1)^{j}\left(\frac{\left(\phi_{R}-1\right)}{\phi_{R}}\right)^{n-1-j}
$$

which can be shown to hold term by term. We have:

$$
\phi\left(\begin{array}{c}
n-1 \\
j
\end{array}\right)(\phi-1)^{j}\left(\phi\left(\phi_{R}-1\right)\right)^{n-1-j}<\phi_{R}^{n-1}\left(\begin{array}{c}
n-1 \\
j
\end{array}\right)(\phi-1)^{j}\left(\frac{\left(\phi_{R}-1\right)}{\phi_{R}}\right)^{n-1-j},
$$

which simplifies to:

$$
\phi^{n-j}<\phi_{R}^{j}
$$

\footnotetext{
${ }^{22}$ Note that for $\phi_{B}>\phi_{3}$, we have $G\left(v \mid z_{B}\right)>0$.
} 


\begin{tabular}{|c|c|c|c|c|c|c|c|c|}
\hline & & \multicolumn{7}{|c|}{ Agents $z_{R}$} \\
\hline & & $r$ & $\mathrm{rb}$ & b & bv & rbv & rv & $\mathbf{v}$ \\
\hline \multirow{7}{*}{ Agents $Z_{B}$} & $r$ & & info & info & info & info & info & info \\
\hline & $\mathrm{rb}$ & BLM & info & info & info & info & info & info \\
\hline & b & BLM & BLM & & info & info & info & info \\
\hline & bv & no veto & BLM & & info & info & info & info \\
\hline & rbv & no veto & info & info & info & info & info & info \\
\hline & rv & FP & info & info & info & info & info & info \\
\hline & v & BLM & Prop 2 & & & Prop 2 & Prop 2 & \\
\hline
\end{tabular}

Figure 2: All possible classes of (symmetric) strategy profiles. Strategy $x y$ for agents of type $z$ means that they play $x$ and $y$ with (strictly) positive probability.

Since we consider here $\phi \leq \phi_{2}=\left(\phi_{R}\right)^{\frac{n+1}{n-1}}$, it is enough to show that $\left(\phi_{R}\right)^{\frac{n+1}{n-1}} \leq\left(\phi_{R}\right)^{\frac{j}{n-j}}$. or, equivalently, that $\frac{n+1}{n-1} \leq \frac{j}{n-j}$. This last equality is satisfied since we are considering $j \geq \frac{n+1}{2}$. And, by continuity of $g(\phi)$, we have that $\phi_{2} \leq \phi_{3} \leq \phi_{4}$.

Finally, note that we prove that $\phi_{3}$ is unique in Part (iv) of the proof of Proposition 2.

\section{No Other Equilibria}

We show that there cannot be other responsive symmetric equilibria than those described in Propositions 2 and 3. The proof is rather straightforward, but quite tedious, so we organize it with the following matrix that considers all the possible classes of (symmetric) strategy profiles. Possible classes of strategy for an agent are given by: $\{r, r b, b, b v, v, b r v, r v\}$, where for instance $r b$ means that this agent plays $r$ and $b$ (but not $v$ ) with strictly positive probability.

We show in five steps that the only possible equilibria correspond to the equilibria described in Propositions 2 (cells "BLM") and 3 (cells "FP").

First, note that if agent $z_{B}$ play $r$ with positive probability at equilibrium, it must be the case that agent $z_{R}$ plays $r$ with probability 1 . This is just because signals are informative. Formally, from equations (7), (5), and (6) we have:

$$
G\left(r \mid z_{B}\right) \geq 0 \Rightarrow G\left(r \mid z_{R}\right)>0
$$

and

$$
G\left(r \mid z_{B}\right) \geq G\left(v \mid z_{B}\right) \Rightarrow G\left(r \mid z_{R}\right)>G\left(v \mid z_{R}\right)
$$

Similarly, if agent $z_{R}$ plays $v$ with positive probability, then agent $z_{B}$ plays $v$ with probability 1 . These two restrictions rule out the cases corresponding to the shaded cells with reference "info".

Second, we can rule out a series of remaining cases where the strategy profile is not responsive. 
These are the cells in dark grey.

Third, from the characterization of the equilibrium in Proposition 2, we have that: (i) if agents $z_{B}$ play $v$ with probability 1 , then agents $z_{R}$ can only be pivotal if all agents receive a signal $z_{R}$. In which case they strictly prefer to play $r$. We can therefore rule out these profiles as well. The corresponding cells are shaded and labelled "Prop 2".

Fourth, from (7), it is easy to show that, if agents $z_{B}$ play $b v$ or $b r v$, then $z_{R}$ plays $r$ with probability 1 only if $\phi_{B} \leq \phi_{2}$. Yet, for $\phi_{B} \leq \phi_{2}$, we can prove from (6) that $G\left(v \mid z_{B}\right)<0$, a contradiction. We can therefore rule out these profiles as well. The corresponding cells are shaded and labelled "no veto".

Finally, the remaining cells correspond to the two equilibria we have characterized. And we have shown that they are both unique within their strategy profile class.

\section{Unanimity}

We characterize the unique responsive equilibrium under Unanimity. Doing so, we extend the equilibrium characterization in Feddersen and Pesendorfer (1998) to biased information structure and the possible presence of a private value agent.

As under voting system $V, z_{P}$-agents strictly prefers to play $v$, i.e. $\sigma_{v}^{U}\left(z_{P}\right)=1$, in any responsive symmetric equilibrium under $U$. It is therefore straightforward to extend Proposition 1 to $U$, and prove that common-value agents always behave as if all agents have common values:

Proposition 6 For any value of $\beta \in[0,1], \sigma^{* U}$ is a responsive symmetric equilibrium under Unanimity if and only if:

i) private-value agents veto the reform,

ii) common-value agents play $\sigma^{0 U}$, where $\sigma^{0 U}$ is a responsive symmetric equilibrium of the game when $\beta=0$.

Proof. Identical to that of Proposition 1.

As for Veto, this Proposition greatly simplifies the equilibrium analyzing by allowing us to focus on the pure common-value game in which $\beta=0$. This is exactly what we do in the remainder of this appendix.

\section{Pivot Probabilities and Expected Payoffs}

Under Unanimity, a vote for $v$ is pivotal if and only if, without that vote, the group decision is $B$ but, with that vote, it becomes $R$. This happens when no other agent is casting a $v$-vote (i.e. $\left.x_{v}=0\right)$. In this case, we say that the vote is pivotal in favor of $R$. As we do under Veto, we denote this event in state $\omega$ by $\operatorname{piv}_{B}^{\omega, U}$. The probability of that event depends on expected vote shares, 
which in turn depends on the state of the world and agent strategies. We denote $\operatorname{Pr}\left(p i v_{B}^{\omega, U}\right)$ the probability to be pivotal in favor of $R$ in state $\omega$ under voting system $U$. Formally:

$$
\operatorname{Pr}\left(\operatorname{piv}_{B}^{\omega, U}\right)=\left(\tau_{r}^{\omega}\right)^{n-1}
$$

A vote for $r$ cannot be pivotal. Indeed, there is no combination of other agents' vote such that the decision is $B$ without this vote and becomes $R$ with it. In fact, either $x_{v}>0$ and the "current" outcome can no longer be changed, or $x_{v}=0$, which implies $x_{r}=n-1>x_{b}=0$, and the "current" outcome is $R$. Finally, a vote for $r$ cannot be pivotal in favor of $B$ as it is a vote against $B$. Remember that $b$ is not an available action under $U$.

From (1), we have that the expected payoff of actions $v$ for an agent of type $z \in\left\{z_{R}, z_{B}\right\}$ under voting system $U$ is

$$
G^{U}(v \mid z)=\operatorname{Pr}\left(\omega_{B} \mid z\right) \operatorname{Pr}\left(p i v_{B}^{\omega_{B}, U}\right)-\operatorname{Pr}\left(\omega_{R} \mid z\right) \operatorname{Pr}\left(p_{i} v_{B}^{\omega_{R}, U}\right)
$$

and the expected payoff of actions $r$ for an agent with signal $z \in\left\{z_{R}, z_{B}\right\}$ under voting system $U$ is

$$
G^{U}(r \mid z)=0
$$

\section{Equilibrium Voting Behavior}

The following proposition extends the equilibrium characterization in Feddersen and Pesendorfer (1998) to possibly biased information structures and the possible presence of a private value voter.

Proposition $\mathbf{7}$ The following strategy profile is the unique responsive symmetric equilibrium under Unanimity:

(i) if $\phi_{B}<\phi_{4}$, then

$$
\begin{aligned}
& \sigma_{r}^{* U}\left(z_{R}\right)=1 \\
& \sigma_{r}^{* U}\left(z_{B}\right)=\alpha^{*}, \text { and } \sigma_{v}^{* U}\left(z_{B}\right)=1-\alpha^{*} .
\end{aligned}
$$

with $\alpha^{*}=\frac{\left(\phi_{B}-1\right)\left(\phi_{R}-\left(\phi_{B}\right)^{\frac{1}{n-1}}\right)}{\left(\phi_{R}-1\right)\left(\left(\phi_{B}\right)^{\frac{1}{n-1}} \phi_{B}-1\right)} \in[0,1)$.

(ii) if $\phi_{B} \geq \phi_{4}$, then

$$
\sigma_{r}^{* U}\left(z_{R}\right)=1 \text {, and } \sigma_{v}^{* U}\left(z_{B}\right)=1 .
$$

Proof. The proof is in two parts. First, we characterize the equilibrium when $\phi_{B} \leq\left(\phi_{R}\right)^{n-1}=\phi_{4}$. Second, we characterize the equilibrium when $\left(\phi_{R}\right)^{n-1}<\phi_{B}$. Proving uniqueness is tedious but straightforward. We therefore omit the details of that part. 
(i): $\phi_{B} \in\left(1, \phi_{4}\right)$. For that set of parameter values, we conjecture that the following strategy profile

$$
\begin{aligned}
& \sigma_{r}^{U}\left(z_{R}\right)=1, \\
& \sigma_{r}^{U}\left(z_{B}\right)=\alpha^{*}, \text { and } \sigma_{v}^{U}\left(z_{B}\right)=1-\alpha^{*},
\end{aligned}
$$

with $\alpha^{*}=\frac{\left(\phi_{B}-1\right)\left(\phi_{R}-\left(\phi_{B}\right)^{\frac{1}{n-1}}\right)}{\left(\phi_{R}-1\right)\left(\left(\phi_{B}\right)^{\frac{1}{n-1}} \phi_{B}-1\right)}$, constitutes an equilibrium.

This requires (i.a) $G\left(v \mid z_{R}\right) \leq 0$, and (i.b) $G\left(v \mid z_{B}\right)=0$. From $G\left(v \mid z_{R}\right) \leq G\left(v \mid z_{B}\right)$ and (i.a), we have that condition (i.b) is necessarily satisfied. From (17), we have that (i.a) is satisfied when:

$$
\operatorname{Pr}\left(\operatorname{piv}_{B}^{\omega_{B}, U}\right)-\phi_{R} \operatorname{Pr}\left(\text { piv }_{B}^{\omega_{R}, U}\right)=0
$$

From (16), and $\tau_{r}^{\omega, U}=\operatorname{Pr}\left(z_{R} \mid \omega\right)+\alpha^{*} \operatorname{Pr}\left(z_{B} \mid \omega\right)$, this boils down to

$$
\left(\frac{\phi_{R}\left(\phi_{B}-1\right)+\alpha^{*}\left(\phi_{R}-1\right)}{\left(\phi_{B}-1\right)+\alpha^{*} \phi_{B}\left(\phi_{R}-1\right)}\right)^{n-1}=\phi_{B}
$$

Therefore, we have that

$$
\alpha^{*}=\frac{\left(\phi_{B}-1\right)\left(\phi_{R}-\left(\phi_{B}\right)^{\frac{1}{n-1}}\right)}{\left(\phi_{R}-1\right)\left(\left(\phi_{B}\right)^{\frac{1}{n-1}} \phi_{B}-1\right)} .
$$

From $\phi_{B} \in\left(1, \phi_{4}\right)$, we have that $\left(\phi_{B}-1\right)\left(\phi_{R}-\left(\phi_{B}\right)^{\frac{1}{n-1}}\right)>0$, which boils down to $\phi_{R}^{n-1}>$ $\phi_{B}$ (satisfied by assumption in the case under consideration). We also have that $\left(\phi_{R}-1\right)\left(\left(\phi_{B}\right)^{\frac{1}{n-1}} \phi_{B}-1\right)>$ 0 . Therefore, $\alpha^{*}>0$. Finally, we have that $\left(\phi_{R}-1\right)\left(\left(\phi_{B}\right)^{\frac{1}{n-1}} \phi_{B}-1\right)>\left(\phi_{B}-1\right)\left(\phi_{R}-\left(\phi_{B}\right)^{\frac{1}{n-1}}\right)$. Therefore $\alpha^{*}<1$.

(ii): $\phi_{4} \leq \phi_{B}$. For that set of parameter values, we conjecture that the following strategy profile

$$
\sigma_{r}\left(z_{R}\right)=1, \text { and } \sigma_{v}\left(z_{B}\right)=1
$$

constitutes an equilibrium. This requires (ii.a) $G\left(v \mid z_{R}\right) \leq 0$, and (ii.b) $G\left(v \mid z_{B}\right) \geq 0$.

First, we discuss condition (ii.a). From (17), we have that condition (ii.a) is satisfied if

$$
\operatorname{Pr}\left(\text { piv }_{B}^{\omega_{B}}\right)-\phi_{R} \operatorname{Pr}\left(\text { piv }_{B}^{\omega_{R}}\right) \leq 0 .
$$

From (16), and $\tau_{r}^{\omega}=\operatorname{Pr}\left(z_{R} \mid \omega\right)$, this boils down to $\left(\phi_{R}\right)^{n} \geq 1$, which is always satisfied.

Second, we discuss condition (ii.b) From (17), we have that condition (ii.b) is satisfied if

$$
\phi_{B} \operatorname{Pr}\left(p i v_{B}^{\omega_{B}}\right)-\operatorname{Pr}\left(p i v_{B}^{\omega_{R}}\right) \geq 0 .
$$


From (16), and $\tau_{r}^{\omega}=p\left(z_{R} \mid \omega\right)$, this boils down to $\phi_{B} \geq\left(\phi_{R}\right)^{n-1}$, which is satisfied by assumption in the case under consideration.

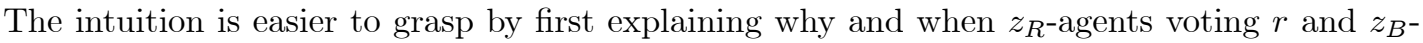
agents voting $v$ is not an equilibrium (i.e. $\phi_{B}<\phi_{4}$ ). Consider an agent who receives signal $z_{B}$. She believes, but is not sure, that $B$ is a better decision than $R$. When deciding which vote to cast, she only focuses on situations in which her vote is pivotal. Under Unanimity, this only happens when all other agents vote for $r$ (event piv $\left.v_{B}^{\omega}\right)$. If $z_{R}$-agents vote $r$ and $z_{B}$-agents vote $v$, this happens if all other agents have received a signal $z_{R}$, which is more likely to happen in state $\omega_{R}$ than in state $\omega_{B}$. As long as the precision of the $z_{B}$ signal is not too high (i.e. $\phi_{B}<\phi_{4}$ ), the joint event ( $n-1 z_{R}$-signals and $1 z_{B}$-signal) is also more likely in state $\omega_{R}$ than in state $\omega_{B}$. She is thus

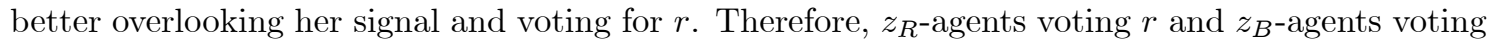
$v$ cannot be an equilibrium in this case.

To understand why the equilibrium is in mixed strategies when $\phi_{B}<\phi_{4}$, notice that when $z_{B}$-agents mix between $r$ and $v$, the information content conditional on being pivotal decreases (since $z_{B}$-agents also vote $r$ with positive probability, the fact of being pivotal no longer hinges on all other agents having received $z_{R}$-signals; hence, the posterior probability of being in state $\omega_{R}$ when being pivotal decreases). For $\sigma_{r}\left(z_{B}\right)$ large enough, this information content is too low to convince $z_{B}$-agents to overlook their signal. The equilibrium corresponds to the case where the posterior probability of being in either state is equal, which makes agents indifferent between the two actions.

For $\phi_{B} \geq \phi_{4}$, the precision of the $z_{B}$ signal is so high that a $z_{B}$-agent remains convinced that state $\omega_{B}$ is more likely when all other agents received a $z_{R}$-signal. In other words, a single $z_{B}$ signal would suffice to convince an agent that could obverse the $n$ signals and could decide for the

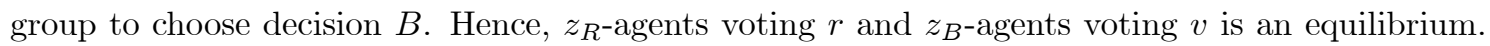
Note that there always is an $n$ sufficiently large such that this case does not arise. 